\title{
A NOVEL LINEAR SECOND ORDER UNCONDITIONALLY ENERGY STABLE SCHEME FOR A HYDRODYNAMIC Q-TENSOR MODEL OF LIQUID CRYSTALS
}

\author{
JIA ZHAO *, XIAOFENG YANG ${ }^{\dagger}$, YUEZHENG GONG ${ }^{\natural}$ AND QI WANG ${ }^{\ddagger}$
}

\begin{abstract}
The hydrodynamic Q-tensor model has been used for studying flows of liquid crystals and liquid crystal polymers. It can be derived from a variational approach together with the generalized Onsager principle, in which the total energy decreases in time. In this paper, we develop a novel, linear, second order semi-discrete scheme in time to solve the governing system. The scheme is developed following the so called ' energy quadratization' strategy so that it is linear and unconditionally energy stable at the semi-discrete level. This scheme is further discretized in space using a second order finite difference method and implemented on a GPU for high performance computing. The convergence rate in time is established using a mesh refinement test. Several numerical examples are presented to demonstrate the usefulness of the model and the effectiveness of the numerical scheme in simulating defect dynamics in flows of liquid crystals.
\end{abstract}

\section{INTRODUCTION}

The liquid crystal is an intermediate phase, which possesses properties of both the liquid and the solid simultaneously. Namely, there exists some partial order either in space (positional order) or in molecular orientation (orientational order). There are several types of liquid crystal phases classified based on their orientational and the positional order, including the nematic phase, the cholesteric phase, the smectic phase, the biaxial phase, and the columnar phase (cf. $[4,6]$ ). Each type requires a specific free energy to characterize its thermodynamic properties and static behavior. There has not been a unified theory that applies to all liquid crystal phases presently. For flows of liquid crystals, hydrodynamics must be coupled with thermodynamic properties. The variational principle together with the generalized Onsager principle has emerged as a new paradigm to derive proper hydrodynamic theories for flows of liquid crystals. Theories so derived normally possess a variational structure as well as the energy dissipation property $[2,9,18,25,26,42,49]$.

There have been two primary types of theories for this flowing material system, each of which targets a different length scale and phenomena. The kinetic theory is built upon a microstructural probability distribution function coupled to a macroscopic flow field. A representative of the kinetic theories is the well-known Doi-Hess theory for flows of nematic liquid crystal polymers (cf. [8]), which describes mesoscopic dynamics of flows of nematic liquid crystals. The other is the continuum model for flows of nematic liquid crystals that we consider in this paper.

In the continuum description for flows of nematic liquid crystals, there exist mainly two descriptions for the average molecular orientation of liquid crystal molecules. One uses a unit vector (known as

Key words and phrases. Q-Tensor Model, Energy Quadratization, Hydrodynamics, Unconditional Energy Stability, Linear, Second Order.

* Department of Mathematics, University of South Carolina, Columbia, SC 29208, USA; Department of Mathematics, University of North Carolina at Chapel Hill, Chapel Hill, NC, 27599; Email: zhaojia@email.unc.edu.

$\dagger$ Department of Mathematics, University of South Carolina, Columbia, SC 29208, USA, Email: xfyang@math.sc.edu.

七 Beijing Computational Science Research Center, Beijing 100931, China; Email: gongyuezheng@csrc.ac.cn.

\$ Beijing Computational Science Research Center, Beijing, 100931, China; Department of Mathematics, IMI and the NanoCenter at USC, University of South Carolina, Columbia, SC 29208; School of Materials Science and Engineering, Nankai University, Tianjin, 300071, China; Email: qwang@csrc.ac.cn.

1

(C) 2016. This manuscript version is made available under the Elsevier user license

http://www.elsevier.com/open-access/userlicense/1.0/ 
the director), that represents the macroscopic orientation of nematic liquid crystals. The representative models are the Ericksen-Leslie model [19] and its extensions to include a scalar order parameter for variable degree of orientation [11]. There have been a quite large number of works on the analysis [3,11,20-22] and numerical study of the models $[1,22,24,49-51]$. The least desirable aspects of the unit vector model is its singularities at point defects and inability to represent line defects as well as biaxiality in the original Ericksen-Leslie theory. An alternative approach is to use a second order tensor $\mathbf{M}$ with $\operatorname{tr}(\mathbf{M})=1$ (i.e. the trace of $\mathbf{M}$ is 1) to describe the macroscopic orientation of liquid crystals, which is identifiable with the second moment of the orientational distribution function of liquid crystals in a kinetic theory [36]. In practice, the deviatoric part of the second moment tensor or the $\mathbf{Q}$-tensor, $\mathbf{Q}=\mathbf{M}-\frac{1}{3} \mathbf{I}$ is often used in place of the second moment tensor $[28,38]$. It measures the deviation of $\mathbf{M}$ from its isotropic value. Here $\mathbf{Q}$ is a symmetric, traceless $3 \times 3$ tensor. It has been established in [38] that the director theories are derivable from Q-tensor theories in the weak flow and weak elastic limit. Thus, it is perceived that director theories are applicable to low molecular weight liquid crystals while the tensor based model can be applied to liquid crystal polymers as well.

In this paper, we focus on a Q-tensor based theory for flows of nematic liquid crystals. To facilitate our presentation, we define two tensor spaces: the symmetric second order tensor space $\mathcal{M}$ and the symmetric and traceless second order tensor space $\mathcal{M}_{t r 0}$,

$$
\begin{aligned}
& \mathcal{M}=\left\{\mathbf{M}: \mathbf{M} \in \mathbb{R}^{3,3}, \mathbf{M}=\mathbf{M}^{T}\right\}, \\
& \mathcal{M}_{t r 0}=\left\{\mathbf{Q}: \mathbf{Q} \in \mathbb{R}^{3,3}, \mathbf{Q}=\mathbf{Q}^{T}, \operatorname{tr}(\mathbf{Q})=0\right\},
\end{aligned}
$$

where $\operatorname{tr}(\mathbf{Q})$ is the trace of tensor $\mathbf{Q}$. We define projection operator $\mathcal{P}: \mathcal{M} \longrightarrow \mathcal{M}_{\text {tro } 0}$ as follows,

$$
\mathcal{P}(\mathbf{M})=\mathbf{M}-\frac{1}{3} \operatorname{tr}(\mathbf{M}), \quad \forall \mathbf{M} \in \mathcal{M} \text {. }
$$

For any $\mathbf{Q} \in \mathcal{M}_{t r 0}$, one can always decompose $\mathbf{Q}$ in its spectral representation

$$
\mathbf{Q}=\sum_{i=1}^{3} \lambda_{i} \mathbf{n}_{i} \mathbf{n}_{i}^{T}
$$

where $\mathbf{n}_{i}, i=1,2,3$ are the unit column eigenvectors of $\mathbf{Q}$, ( which forms an orthonormal basis in $\mathbb{R}^{3}$ ), and $\lambda_{i}$ are the corresponding eigenvalues. Since $\operatorname{tr}(\mathbf{Q})=0$, one deduces $\sum_{i=1}^{3} \lambda_{i}=0$. In addition for liquid crystal, $-\frac{1}{3} \mathbf{I} \leqslant \mathbf{Q} \leqslant \frac{2}{3} \mathbf{I}$ where $\mathbf{A} \leqslant \mathbf{B}$ means $\mathbf{B}-\mathbf{A}$ is a positive definite matrix [36], i.e. $-\frac{1}{3} \leqslant \lambda_{i} \leqslant \frac{2}{3}$. If $\lambda_{i} \neq \lambda_{j}, i \neq j$, then $\mathbf{Q}$ is called biaxial, otherwise $\mathbf{Q}$ is called uniaxial. So, the $\mathbf{Q}$ tensor model can describe both uniaxiality and biaxiality in flows of nematic liquid crystals while the director model such as the Ericksen-Leslie model can only handle uniaxiality.

For nematic liquid crystals, we consider the following free energy in the Q tensor model

$$
\mathbb{E}(\mathbf{Q})=\int_{\Omega}\left(\frac{L}{2}|\nabla \mathbf{Q}|^{2}+F_{B}(\mathbf{Q})\right) d \boldsymbol{x},
$$

where $\Omega$ is the material domain, $L$ is an elastic constant and $F_{B}(\mathbf{Q})$ is the bulk free energy density, given by

$$
F_{B}(\mathbf{Q})=\frac{a}{2} \operatorname{tr}\left(\mathbf{Q}^{2}\right)-\frac{b}{3} \operatorname{tr}\left(\mathbf{Q}^{3}\right)+\frac{c}{4} \operatorname{tr}^{2}\left(\mathbf{Q}^{2}\right),
$$

where $a, b, c$ are constants, $c>0$ is usually assumed so that $F_{B}(\mathbf{Q})$ can attains a minimum which is physically required.

Let $\mathbf{D}$ denote the rate of strain tensor and $\mathbf{W}$ the vorticity tensor as

$$
\mathbf{D}=\frac{1}{2}\left(\nabla \mathbf{u}+\nabla \mathbf{u}^{T}\right), \quad \mathbf{W}=\frac{1}{2}\left(\nabla \mathbf{u}-\nabla \mathbf{u}^{T}\right)
$$


with $(\nabla \mathbf{u})_{i j}=\partial_{j} \mathbf{u}_{i}, i, j=1,2,3$. The nondimensionalized hydrodynamic $\mathbf{Q}$-tensor model of nematic liquid crystals is summarized as follows (cf. $[2,37,47]$ ),

$$
\left\{\begin{array}{l}
\rho\left(\mathbf{u}_{t}+\mathbf{u} \cdot \nabla \mathbf{u}\right)=-\nabla p+\eta \Delta \mathbf{u}+\nabla \cdot \boldsymbol{\Sigma}-\boldsymbol{H} \nabla \mathbf{Q} \\
\nabla \cdot \mathbf{u}=0 \\
\mathbf{Q}_{t}+\mathbf{u} \cdot \nabla \mathbf{Q}-\boldsymbol{S}=M \boldsymbol{H}
\end{array}\right.
$$

with $(\boldsymbol{H} \nabla \mathbf{Q})_{k}=\boldsymbol{H}_{i j} \nabla_{k} \mathbf{Q}_{i j}$, and

$$
\begin{aligned}
& \boldsymbol{H}=-\mathcal{P}\left(\frac{\delta \mathbb{E}}{\delta \mathbf{Q}}\right)=L \Delta \mathbf{Q}-a \mathbf{Q}+b\left(\mathbf{Q}^{2}-\frac{1}{3} \operatorname{tr}\left(\mathbf{Q}^{2}\right) \mathbf{I}\right)-c \operatorname{tr}\left(\mathbf{Q}^{2}\right) \mathbf{Q}, \\
& \boldsymbol{S}=\mathbf{W} \cdot \mathbf{Q}-\mathbf{Q} \cdot \mathbf{W}+\xi(\mathbf{Q} \cdot \mathbf{D}+\mathbf{D} \cdot \mathbf{Q})+\frac{2 \xi}{3} \mathbf{D}-2 \xi(\mathbf{D}: \mathbf{Q})\left(\mathbf{Q}+\frac{1}{3} \mathbf{I}\right), \\
& \boldsymbol{\Sigma}=(\mathbf{Q} \cdot \boldsymbol{H}-\boldsymbol{H} \cdot \mathbf{Q})-\xi(\boldsymbol{H} \cdot \mathbf{Q}+\mathbf{Q} \cdot \boldsymbol{H})-\frac{2 \xi}{3} \boldsymbol{H}+2 \xi(\mathbf{Q}: \boldsymbol{H})\left(\mathbf{Q}+\frac{1}{3} \mathbf{I}\right),
\end{aligned}
$$

where $\rho$ is the dimensionless density which is normally set to $\rho=1, \boldsymbol{H}$ is the molecular field, the first two terms in $\boldsymbol{S}$ together with the material derivative of $\mathbf{Q}$ defines the Gordon-Schowalter derivative, $p$ is the hydrostatic pressure, $\boldsymbol{\Sigma}$ is the elastic stress, $1 / M$ is the relaxation time, $\xi$ is a geometric parameter of the nematic liquid crystal molecule that is confined between -1 and 1 (cf. [37]). In the following, we work with the dimensionless equations.

The initial conditions of the system are

$$
\mathbf{u}(t=0)=\mathbf{u}_{0}, \mathbf{Q}(t=0)=\mathbf{Q}_{0} .
$$

For the boundary conditions, $\mathbf{u}$ and $\mathbf{Q}$ are either

(i) periodic on $\partial \Omega$; or

(ii) $\left.\mathbf{u}\right|_{\partial \Omega}=0,\left.\mathbf{Q}\right|_{\partial \Omega}=\mathbf{Q}^{0}$, or $\left.\partial_{n} \mathbf{Q}\right|_{\partial \Omega}=0$,

where $\mathbf{Q}_{0}$ and $\mathbf{Q}^{0}$ are prescribed tensor functions as the initial and boundary condition of the problem, respectively.

The hydrodynamic $\mathbf{Q}$-tensor model has been studied in various flow geometries like shear and elongation flows to predict rheological properties of nematic liquid crystal polymers $[2,7,30,36,37]$. For some analytical and numerical works related with this model, we refer to $[3,13,23,27,29]$ and the references therein. Despite that some numerical codes are developed to simulate flow behavior of nematic liquid crystals using the model, there have never been a systematic effort to analyze the numerical scheme used for solving the model equations exploring the variational and dissipative property of the model (1.7). As we will show in the next section that the governing system of equations (1.7) obeys a total energy dissipation law. It would therefore be desirable to develop numerical schemes that respect the energy dissipation property. This type numerical scheme is called the energy stable scheme. The challenge to developing efficient numerical schemes while preserving energy stability for this model lies in (i) the bulk potential (1.5) is highly nonlinear, and (ii) there exists a strong coupling between the velocity field $\mathbf{u}$ and tensor field $\mathbf{Q}$, in the nonlinear stress term $\boldsymbol{\Sigma}$ (defined in (1.10)) and the term $\boldsymbol{S}$ (defined in (1.9)). In [47], we have designed a first and a second order semi-discrete scheme in time employing both the convex-splitting strategy $[12,31,40]$ and the stabilizer approach $[32,33]$, and showed the developed numerical schemes are unconditionally energy stable. However, the second order scheme is nonlinear and implicit, which can potentially lead to difficulties in numerical implementation and normally requires nontrivial iterations.

Recently, we have developed a general strategy to transform a series of nonlinear bulk potentials into quadratic forms via the change of variables in $[43-46,48]$ such that one can readily obtain a linear numerical scheme for various stiff dissipative systems that involve nonlinear potentials in complex formats. We coined a name for the practice: energy quadratization. In this paper, we will follow the energy quadratization strategy to develop numerical schemes to solve the governing system of equations in the 
Q-tensor model. The obtained new semi-discrete scheme is not only linear and second order accurate, but also unconditionally energy stable in the sense that it maintains energy dissipation at the semidiscrete level irrespective of the time step in the discretization. This novel energy quadratization strategy can be readily adapted to develop linear, stable numerical schemes for models derived via total energy variation $[10,35,39-41]$.

In the computer implementation of the numerical scheme, we discretize the temporal semi-discrete scheme in space using a second order finite difference method subject to the given boundary condition. We then implement the second order, linear, energy stable scheme on a GPU card to enhance computational efficiency. We first benchmark the convergence rate numerically through a mesh refinement test and then study four different examples with a focus on defect dynamics in flows of nematic liquid crystals. These include (i) how defects of the same degree separate from each other in the case of Dirichlet boundary conditions for the $\mathbf{Q}$ tensor; (ii) how hydrodynamics affect defect dynamics in the case of defect nucleation and annihilation; (iii) how boundary conditions dictate defect dynamics; and (iv) how shear flow affects defect dynamics in a cavity flow geometry. These numerical examples are presented to demonstrate the effectiveness of the numerical scheme in revealing the intricate dynamics of liquid crystals with defects.

The rest of the paper is organized as follows. In Section 2, a new equivalent reformation of the hydrodynamic Q-tensor model is presented based on the energy quadratization strategy. In Section 3 , a second order semi-discrete, linear scheme in time is presented, where we show rigorously that this new scheme satisfies the discrete energy dissipation law. In Section 4, various numerical examples are presented to validate the accuracy and stability of the scheme. Finally, we give a concluding remark in Section 5 .

\section{Model ReFormulation}

We now reformulate model (1.7) into an equivalent form using the energy quadratization technique developed recently $[16,43-46,48]$, from which we develop novel numerical schemes to solve the governing system of equations in the $\mathbf{Q}$-tensor model. Before proceeding, we introduce some notations that will be used throughout the paper.

For any tensor functions $\mathbf{M}_{1}, \mathbf{M}_{2} \in R^{3 \times 3}$, vector functions $\mathbf{u}, \mathbf{v} \in \mathbb{R}^{3}$ and scalar functions $f, g \in \mathbb{R}$ in $L^{2}(\Omega)$ space, where $\Omega$ is the domain of the functions, we define the following $L^{2}$ inner products:

$$
\left(\mathbf{M}_{1}, \mathbf{M}_{2}\right)=\int_{\Omega} \sum_{i, j=1}^{3}\left(\mathbf{M}_{1}\right)_{i j}\left(\mathbf{M}_{2}\right)_{i j} d \boldsymbol{x}, \quad(\mathbf{u}, \mathbf{v})=\int_{\Omega} \sum_{i=1}^{3} \mathbf{u}_{i} \mathbf{v}_{i} d \boldsymbol{x}, \quad(f, g)=\int_{\Omega} f g d \boldsymbol{x} .
$$

Consequently, the $L^{2}$ norms are given by

$$
\|\mathbf{M}\|^{2}=(\mathbf{M}, \mathbf{M}), \quad\|\mathbf{u}\|^{2}=(\mathbf{u}, \mathbf{u}), \quad\|f\|^{2}=(f, f) .
$$

We establish the following bounded property for the bulk potential $F_{B}(\mathbf{Q})$.

Theorem 2.1. Given any constant $c>0$, the bulk potential

$$
F_{B}(\mathbf{Q})=\frac{a}{2} \operatorname{tr}\left(\mathbf{Q}^{2}\right)-\frac{b}{3} \operatorname{tr}\left(\mathbf{Q}^{3}\right)+\frac{c}{4} \operatorname{tr}^{2}\left(\mathbf{Q}^{2}\right), \quad \forall \mathbf{Q} \in \mathcal{M}_{t r 0}
$$

has a uniform lower bound that only depends on $a, b, c$.

Proof. We denote the three eigenvalues of $\mathbf{Q}$ by $\lambda_{1}, \lambda_{2}, \lambda_{3}$. Then

$$
\begin{aligned}
F_{B}(\mathbf{Q}) & =\frac{a}{2} \operatorname{tr}\left(\mathbf{Q}^{2}\right)-\frac{b}{3} \operatorname{tr}\left(\mathbf{Q}^{3}\right)+\frac{c}{4} \operatorname{tr}^{2}\left(\mathbf{Q}^{2}\right) \\
& =\frac{a}{2} \sum_{i=1}^{3} \lambda_{i}^{2}-\frac{b}{3} \sum_{i=1}^{3} \lambda_{i}^{3}+\frac{c}{4}\left(\sum_{i=1}^{3} \lambda_{i}^{2}\right)^{2},
\end{aligned}
$$

subject to the constraint $\sum_{i=1}^{3} \lambda_{i}=0$ as $\mathbf{Q} \in \mathcal{M}_{t r 0}$. Without loss of generality, we consider a larger domain for $\left(\lambda_{1}, \lambda_{2}, \lambda_{3}\right) \in \mathbb{R}^{3}$. 
We denote $L_{0}=\max \left(\left|\frac{4 b}{3 c}\right|, \sqrt{\left|\frac{a}{c}\right|}\right)$. In the closed domain $\left[-L_{0}, L_{0}\right]^{3}$, since $F_{B}(\mathbf{Q})$ is continuous, it has a global minimum, say

$$
\min _{\left(\lambda_{1}, \lambda_{2}, \lambda_{3}\right) \in\left[-L_{0}, L_{0}\right]^{3}} F_{B}(\mathbf{Q})=C_{0}
$$

In the domain $\left(\left(-\infty,-L_{0}\right] \cup\left[L_{0}, \infty\right)\right)^{3}$, we easily show that

$$
F_{B}(\mathbf{Q})=\sum_{i=1}^{3}\left(\frac{c}{4} \lambda_{i}^{2}-\frac{b}{3} \lambda_{i}\right) \lambda_{i}^{2}+\frac{c}{2}\left(\lambda_{1}^{2}\left(\lambda_{2}^{2}+\frac{a}{c}\right)+\lambda_{2}^{2}\left(\lambda_{3}^{2}+\frac{a}{c}\right)+\lambda_{3}^{2}\left(\lambda_{1}^{2}+\frac{a}{c}\right)\right) \geqslant 0 .
$$

Therefore, $F_{B}(\mathbf{Q}) \geqslant \min \left(0, C_{0}\right), \forall \mathbf{Q} \in \mathbb{R}^{3}$, i.e., $F_{B}(\mathbf{Q})$ has a uniform lower bound that only depends on $a, b, c$.

By Theorem 2.1, for any given parameters $a, b, c(c>0)$, we can always pick a constant $A_{0}$, that only depends on $a, b, c$, such that $F_{B}(\mathbf{Q})+A_{0}>0$. Then, we introduce a new scalar variable $q$ defined by

$$
q(\mathbf{Q})=\sqrt{2\left(\frac{a}{2} \operatorname{tr}\left(\mathbf{Q}^{2}\right)-\frac{b}{3} \operatorname{tr}\left(\mathbf{Q}^{3}\right)+\frac{c}{4} \operatorname{tr}^{2}\left(\mathbf{Q}^{2}\right)+A_{0}\right)}
$$

It follows that

$$
\frac{\delta q}{\delta \mathbf{Q}}=\frac{a \mathbf{Q}-b \mathbf{Q}^{2}+c \operatorname{tr}\left(\mathbf{Q}^{2}\right) \mathbf{Q}}{\sqrt{2\left(\frac{a}{2} \operatorname{tr}\left(\mathbf{Q}^{2}\right)-\frac{b}{3} \operatorname{tr}\left(\mathbf{Q}^{3}\right)+\frac{c}{4} \operatorname{tr}^{2}\left(\mathbf{Q}^{2}\right)+A_{0}\right)}} .
$$

In terms of the new symbol $q$, we rewrite the free energy as follows:

$$
E(\mathbf{Q}, q)=\int_{\Omega}\left(\frac{L}{2}|\nabla \mathbf{Q}|^{2}+\frac{1}{2} q^{2}-A_{0}\right) d \boldsymbol{x}
$$

We further denote

$$
B(\mathbf{u}, \mathbf{v})=\mathbf{u} \cdot \nabla \mathbf{v}+\frac{1}{2}(\nabla \cdot \mathbf{u}) \mathbf{v}
$$

One can easily see that $B(\mathbf{u}, \mathbf{u})=\mathbf{u} \cdot \nabla \mathbf{u}$ when $\nabla \cdot \mathbf{u}=0$. The extra term $\frac{1}{2}(\nabla \cdot \mathbf{u}) \mathbf{v}$ in $B(\mathbf{u}, \mathbf{v})$ was first introduced in [34]. Then, we have the broadly used property as follows (cf. [5, 17])

Lemma 2.1. Given the trilinear form,

$$
\widehat{B}(\mathbf{u}, \mathbf{v}, \mathbf{w})=(B(\mathbf{u}, \mathbf{v}), \mathbf{w}), \quad \forall \mathbf{u}, \mathbf{v}, \mathbf{w} \in H^{1}(\Omega)^{d},
$$

it follows that

$$
\widehat{B}(\mathbf{u}, \mathbf{v}, \mathbf{v})=0
$$

if $\mathbf{u}, \mathbf{v}$ are periodic or satisfy

$$
\mathbf{u} \in H_{0}^{1}(\Omega)^{d}=H^{1}(\Omega)^{d} \cap\left\{\left.\mathbf{u} \cdot \mathbf{n}\right|_{\partial \Omega}=0\right\}, \mathbf{v} \in H^{1}(\Omega)^{d},
$$

where $d$ is the dimension of the domain $\Omega$.

Using the above notations, we rewrite the dynamic equations in (1.7) into the following form,

$$
\left\{\begin{array}{l}
\mathbf{u}_{t}+B(\mathbf{u}, \mathbf{u})=-\nabla p+\eta \nabla^{2} \mathbf{u}+\nabla \cdot \boldsymbol{\sigma}-\mathcal{H} \nabla \mathbf{Q} \\
\nabla \cdot \mathbf{u}=0 \\
\mathbf{Q}_{t}+\mathbf{u} \cdot \nabla \mathbf{Q}-\boldsymbol{S}=M \mathcal{H} \\
q_{t}=\boldsymbol{P}(\mathbf{Q}): \mathbf{Q}_{t}
\end{array}\right.
$$


where

$$
\left\{\begin{array}{l}
\boldsymbol{P}(\mathbf{Q}):=\mathcal{P}\left(\frac{\delta q}{\delta \mathbf{Q}}\right)=\frac{a \mathbf{Q}-b\left(\mathbf{Q}^{2}-\frac{1}{3} \operatorname{tr}\left(\mathbf{Q}^{2}\right) \mathbf{I}\right)+c \operatorname{tr}\left(\mathbf{Q}^{2}\right) \mathbf{Q}}{\sqrt{2\left(\frac{a}{2} \operatorname{tr}\left(\mathbf{Q}^{2}\right)-\frac{b}{3} \operatorname{tr}\left(\mathbf{Q}^{3}\right)+\frac{c}{4} \operatorname{tr}^{2}\left(\mathbf{Q}^{2}\right)+A_{0}\right)}}, \\
\mathcal{H}=-\mathcal{P}\left(\frac{\delta E(\mathbf{Q}, q)}{\delta \mathbf{Q}}\right)=L \Delta \mathbf{Q}-q \boldsymbol{P}(\mathbf{Q}), \\
\boldsymbol{\sigma}(\mathbf{Q}, \mathcal{H})=(\mathbf{Q} \cdot \mathcal{H}-\mathcal{H} \cdot \mathbf{Q})-\xi(\mathcal{H} \cdot \mathbf{Q}+\mathbf{Q} \cdot \mathcal{H})-\frac{2 \xi}{3}\left(\mathcal{H}-\frac{1}{3} \operatorname{tr}(\mathcal{H}) \mathbf{I}\right)+2 \xi(\mathbf{Q}: \mathcal{H}) \mathbf{Q}
\end{array}\right.
$$

where we use slightly different notations $\mathcal{H}$ and $\sigma$ to present $\mathbf{H}$ and $\Sigma$ after the new intermediate variable $\mathbf{q}$ is introduced. They are identical in the PDE system. Notice that in (2.14), no boundary condition for $q$ is necessary since the equation for $q$ is only ODE with time, thus it only needs an initial condition at every $\mathbf{x}$ :

$$
q(t=0)=\sqrt{2\left(\frac{a}{2} \operatorname{tr}\left(\mathbf{Q}_{0}^{2}\right)-\frac{b}{3} \operatorname{tr}\left(\mathbf{Q}_{0}^{3}\right)+\frac{c}{4} \operatorname{tr}^{2}\left(\mathbf{Q}_{0}^{2}\right)+A_{0}\right)} .
$$

The boundary conditions of $\mathbf{u}$ and $\mathbf{Q}$ are given in (1.12).

Before we derive the energy dissipation law for the PDE system (1.7) or its equivalent system (2.14), the following lemma is needed.

Lemma 2.2. For any $\mathbf{u} \in H_{0}^{1}(\Omega)^{d}$, we have

$$
(\mathbf{u}, \nabla \cdot \boldsymbol{\Sigma})=(\boldsymbol{H}, \mathbf{S})
$$

Proof. From (1.9), we have

$$
(\boldsymbol{H}, \mathbf{S})
$$

From (1.10), we have

$$
\begin{aligned}
(\nabla \cdot \boldsymbol{\Sigma}, \mathbf{u}) & =(\nabla \cdot(\boldsymbol{\Sigma} \mathbf{u}), 1)-(\boldsymbol{\Sigma}, \nabla \mathbf{u}) \\
& =\left(-(\mathbf{Q} \cdot \boldsymbol{H}-\boldsymbol{H} \cdot \mathbf{Q})+\xi(\boldsymbol{H} \cdot \mathbf{Q}+\mathbf{Q} \cdot \boldsymbol{H})+\frac{2 \xi}{3} \boldsymbol{H}-2 \xi(\mathbf{Q}: \boldsymbol{H}) \mathbf{Q}, \nabla \mathbf{u}\right),
\end{aligned}
$$

provided $\mathbf{u} \in H_{0}^{1}(\Omega)^{d}$.

Notice that

$$
\begin{aligned}
& -(\mathbf{Q} \cdot \boldsymbol{H}-\boldsymbol{H} \cdot \mathbf{Q}, \nabla \mathbf{u})=(\boldsymbol{H}, \mathbf{W} \cdot \mathbf{Q}-\mathbf{Q} \cdot \mathbf{W}) \\
& \xi(\boldsymbol{H} \cdot \mathbf{Q}+\mathbf{Q} \cdot \boldsymbol{H}, \nabla \mathbf{u})=\xi(\boldsymbol{H}, \mathbf{D} \cdot \mathbf{Q}+\mathbf{Q} \cdot \mathbf{D}) \\
& 2 \xi((\mathbf{Q}: \boldsymbol{H}) \mathbf{Q}, \nabla \mathbf{u})=2 \xi\left(\boldsymbol{H},(\mathbf{Q}: \mathbf{D})\left(\mathbf{Q}+\frac{1}{3} \mathbf{I}\right)\right) \\
& \frac{2 \xi}{3}(\boldsymbol{H}, \nabla \mathbf{u})=\frac{2 \xi}{3}(\boldsymbol{H}, \mathbf{D}) .
\end{aligned}
$$

The combination of the left hand side of $(2.20)$ gives $(\nabla \cdot \boldsymbol{\Sigma}, \mathbf{u})$; analogously, the combination of the right hand side of (2.20) gives $(\boldsymbol{H}, \boldsymbol{S})$. This completes the proof.

We establish the energy law for the system (1.7) as follows.

Theorem 2.2. The total energy in model (1.7) satisfies the following dissipation law

$$
\frac{d}{d t} \mathbb{E}(\mathbf{u}, \mathbf{Q})=-\eta\|\nabla \mathbf{u}\|^{2}-M\|\boldsymbol{H}\|^{2}
$$


where

$$
\mathbb{E}(\mathbf{u}, \mathbf{Q})=\int_{\Omega}\left(\frac{1}{2}|\mathbf{u}|^{2}+\frac{L}{2}|\nabla \mathbf{Q}|^{2}+\frac{a}{2} \operatorname{tr}\left(\mathbf{Q}^{2}\right)-\frac{b}{3} \operatorname{tr}\left(\mathbf{Q}^{3}\right)+\frac{c}{4} \operatorname{tr}^{2}\left(\mathbf{Q}^{2}\right)\right) d \boldsymbol{x} .
$$

Proof. We calculate the energy dissipation rate for (1.7) directly. It follows from the chain rule that

$$
\frac{d}{d t} \mathbb{E}(\mathbf{u}, \mathbf{Q})=\int_{\Omega}\left(\frac{\delta \mathbb{E}}{\delta \mathbf{u}} \mathbf{u}_{t}+\mathcal{P}\left(\frac{\delta \mathbb{E}}{\delta \mathbf{Q}}\right): \mathbf{Q}_{t}\right) d \boldsymbol{x} . .
$$

Notice that $\frac{\delta \mathbb{E}(\mathbf{u}, \mathbf{Q})}{\delta \mathbf{u}}=\mathbf{u}, \mathcal{P}\left(\frac{\delta \mathbb{E}(\mathbf{u}, \mathbf{Q})}{\delta \mathbf{Q}}\right)=-\boldsymbol{H}$ and use (2.14), we then have

$$
\begin{aligned}
\frac{d}{d t} \mathbb{E}(\mathbf{u}, \mathbf{Q})= & (\mathbf{u},-B(\mathbf{u}, \mathbf{u})-\nabla p+\eta \Delta \mathbf{u}+\nabla \cdot \boldsymbol{\Sigma}-\boldsymbol{H} \nabla \mathbf{Q}) \\
& +(-\boldsymbol{H},-\mathbf{u} \cdot \nabla \mathbf{Q}+\boldsymbol{S}+M \boldsymbol{H}) .
\end{aligned}
$$

Applying Lemma 2.1 and Lemma 2.2 and noticing that $(\mathbf{u},-\nabla p)=(\nabla \cdot \mathbf{u}, p)=0$, we arrive at $(2.21)$.

Since the new model (2.14) and model (1.7) are identical, they satisfy the same energy dissipation law as in $(2.21)$, but in terms of the new variable $q$, it reads as follows

$$
\frac{d}{d t} E(\mathbf{u}, \mathbf{Q}, q)=-\eta\|\nabla \mathbf{u}\|^{2}-M\|\mathcal{H}\|^{2} \leqslant 0
$$

where

$$
E(\mathbf{u}, \mathbf{Q}, q)=\int_{\Omega}\left(\frac{1}{2}|\mathbf{u}|^{2}+\frac{L}{2}|\nabla \mathbf{Q}|^{2}+\frac{1}{2} q^{2}-A_{0}\right) d \boldsymbol{x}
$$

\section{Numerical Schemes}

In this section, we develop time-marching numerical schemes for the newly transformed system (2.14). Let $\delta t>0$ be the time step size and $t^{n}=n \delta t$ for $0 \leqslant n$. We denote the discrete value of the variable $(\bullet)(\mathbf{x}, t)$ at $t^{n}$ as $(\bullet)^{n}(\mathbf{x})$, where $(\bullet)$ denotes a generic variable. We next present a linear, second order, semi-discrete scheme in time and show that the new scheme is unconditionally energy stable. To simplify the presentation,we introduce the following notations,

$$
\begin{array}{lll}
(\cdot)^{n+\frac{1}{2}}=\frac{1}{2}\left((\cdot)^{n+1}+(\cdot)^{n}\right), & (\cdot)_{*}^{n+\frac{1}{2}}=\frac{1}{2}\left((\cdot)^{n+1}+(\cdot)^{n}\right), \\
\overline{(\cdot)}^{n+1}=2(\cdot)^{n}-(\cdot)^{n-1}, & \overline{(\cdot)^{n+\frac{1}{2}}}=\frac{3}{2}(\cdot)^{n}-\frac{1}{2}(\cdot)^{n-1},
\end{array}
$$

where $(\because)^{n+1}$ means an intermediate approximation of $(\cdot)$.

3.1. Scheme for the hydrodynamic coupled model. The second order, linear numerical scheme for the hydrodynamic coupled model (2.14) is given in the following.

Scheme 1. Given the initial values of $\left(\mathbf{u}^{0}, p^{0}, \mathbf{Q}^{0}, q^{0}\right)$, we set $\left(\mathbf{u}^{-1}, p^{-1}, \mathbf{Q}^{-1}, q^{-1}\right)=\left(\mathbf{u}^{0}, p^{0}, \mathbf{Q}^{0}, q^{0}\right)$. Then for any $n \geqslant 0$, we obtain $\left(\mathbf{u}^{n+1}, p^{n+1}, \mathbf{Q}^{n+1}, q^{n+1}\right)$ through the following two steps:

Step 1: we solve for $\left(\widetilde{\mathbf{u}}^{n+1}, \mathbf{Q}^{n+1}, q^{n+1}\right)$ using

$$
\left\{\begin{array}{l}
\frac{\widetilde{\mathbf{u}}^{n+1}-\mathbf{u}^{n}}{\delta t}+B\left(\overline{\mathbf{u}}^{n+\frac{1}{2}}, \mathbf{u}_{*}^{n+\frac{1}{2}}\right)=-\nabla p^{n}+\eta \nabla^{2} \mathbf{u}_{*}^{n+\frac{1}{2}}+\nabla \cdot \boldsymbol{\sigma}^{n+\frac{1}{2}}-\mathcal{H}^{n+\frac{1}{2}} \nabla \overline{\mathbf{Q}}^{n+\frac{1}{2}}, \\
\frac{\mathbf{Q}^{n+1}-\mathbf{Q}^{n}}{\delta t}+\mathbf{u}_{*}^{n+\frac{1}{2}} \cdot \nabla \overline{\mathbf{Q}}^{n+\frac{1}{2}}-\boldsymbol{S}^{n+\frac{1}{2}}=M \mathcal{H}^{n+\frac{1}{2}}, \\
q^{n+1}-q^{n}=\overline{\boldsymbol{P}}^{n+\frac{1}{2}}:\left(\mathbf{Q}^{n+1}-\mathbf{Q}^{n}\right),
\end{array}\right.
$$


where

$$
\left\{\begin{array}{l}
\boldsymbol{S}^{n+\frac{1}{2}}=\boldsymbol{S}\left(\nabla \mathbf{u}_{*}^{n+\frac{1}{2}}, \overline{\mathbf{Q}}^{n+\frac{1}{2}}\right), \\
\boldsymbol{\sigma}^{n+\frac{1}{2}}=\boldsymbol{\sigma}\left(\overline{\mathbf{Q}}^{n+\frac{1}{2}}, \mathcal{H}^{n+\frac{1}{2}}\right), \\
\overline{\boldsymbol{P}}^{n+\frac{1}{2}}=\boldsymbol{P}\left(\overline{\mathbf{Q}}^{n+\frac{1}{2}}\right), \\
\mathcal{H}^{n+\frac{1}{2}}=L \Delta \mathbf{Q}^{n+\frac{1}{2}}-q^{n+\frac{1}{2}} \overline{\boldsymbol{P}}^{n+\frac{1}{2}} .
\end{array}\right.
$$

and the boundary conditions are either

(i) periodic; or (ii) $\left.\widetilde{\mathbf{u}}^{n+1}\right|_{\partial \Omega}=0,\left.\mathbf{Q}^{n+1}\right|_{\partial \Omega}=\mathbf{Q}^{0}$ or $\left.\partial_{\mathbf{n}} \mathbf{Q}^{n+1}\right|_{\partial \Omega}=0$.

Step 2: we solve for $\left(\mathbf{u}^{n+1}, p^{n+1}\right)$ using

$$
\left\{\begin{array}{l}
\frac{\mathbf{u}^{n+1}-\widetilde{\mathbf{u}}^{n+1}}{\delta t}=-\frac{1}{2} \nabla\left(p^{n+1}-p^{n}\right), \\
\nabla \cdot \mathbf{u}^{n+1}=0 .
\end{array}\right.
$$

where the boundary conditions are either (i) periodic or (ii) $\left.\mathbf{u}^{n+1} \cdot \mathbf{n}\right|_{\partial \Omega}=0$.

Notice that from (3.2), we can express $q^{n+\frac{1}{2}}$ fas

$$
q^{n+\frac{1}{2}}=\frac{1}{2} \overline{\boldsymbol{P}}^{n+\frac{1}{2}}: \mathbf{Q}^{n+1}-\frac{1}{2} \overline{\boldsymbol{P}}^{n+\frac{1}{2}}: \mathbf{Q}^{n}+q^{n} .
$$

Then we can substitute it into the equation for $\mathcal{H}^{n+1 / 2}$ in (3.3). As a result, the equation for $q^{n+1}$ is decoupled from the others. Thus we can solve for $\left(\mathbf{Q}^{n+1}, \widetilde{\mathbf{u}}^{n+1}\right)$ firstly and then $q^{n+1}$ in step 1 . Specifically, the transport equation for $\mathbf{Q}^{n+1}$ in the scheme can be reformulated as follows

$$
\mathbb{A}\left(\mathbf{Q}^{n+1}, \widetilde{\mathbf{u}}^{n+1}\right)=\mathbb{G}\left(\mathbf{Q}^{n}\right),
$$

where

$$
\begin{aligned}
\mathbb{A}\left(\mathbf{Q}^{n+1}, \widetilde{\mathbf{u}}^{n+1}\right)= & \frac{1}{\delta t} \mathbf{Q}^{n+1}-\frac{M L}{2} \Delta \mathbf{Q}^{n+1}+\frac{M}{2}\left(\overline{\boldsymbol{P}}^{n+\frac{1}{2}}: \mathbf{Q}^{n+1}\right) \overline{\boldsymbol{P}}^{n+\frac{1}{2}} \\
& +\mathbf{u}_{*}^{n+\frac{1}{2}} \cdot \nabla \overline{\mathbf{Q}}^{n+\frac{1}{2}}-\boldsymbol{S}^{n+\frac{1}{2}}, \\
= & \frac{\mathbf{Q}^{n}}{\delta t}+\frac{M L}{2} \Delta \mathbf{Q}^{n}+\frac{M}{2}\left(\overline{\boldsymbol{P}}^{n+\frac{1}{2}}: \mathbf{Q}^{n}\right) \overline{\boldsymbol{P}}^{n+\frac{1}{2}}-M q^{n} \overline{\boldsymbol{P}}^{n+\frac{1}{2}} .
\end{aligned}
$$

We need to prove scheme (3.7) is a linear mapping from $\mathcal{M}_{t r 0} \rightarrow \mathcal{M}_{\text {tr } 0}$. as follows.

Theorem 3.1. Given $\mathbf{Q}^{n-1}, \mathbf{Q}^{n} \in \mathcal{M}_{\text {tr } 0}$, The scheme (3.7) (or (3.2)) warrants that $\mathbf{Q}^{n+1} \in \mathcal{M}_{\text {tr } 0}$.

Proof. Taking the trace for the discrete transport equation of $\mathbf{Q}^{n+1}$ in (3.7), we obtain

$$
\frac{1}{\delta t} \operatorname{tr}\left(\mathbf{Q}^{n+1}\right)-\frac{M L}{2} \Delta \operatorname{tr}\left(\mathbf{Q}^{n+1}\right)+\mathbf{u}_{*}^{n+\frac{1}{2}} \cdot \nabla \operatorname{tr}\left(\overline{\mathbf{Q}}^{n+\frac{1}{2}}\right)-\operatorname{tr}\left(\boldsymbol{S}^{n+\frac{1}{2}}\right)=\operatorname{tr}\left(\mathbb{G}\left(\mathbf{Q}^{n}\right)\right)=0 .
$$

Given that $\mathbf{Q}^{n-1}, \mathbf{Q}^{n} \in \mathcal{M}_{t r 0}$, we derive

$$
\operatorname{tr}\left(\boldsymbol{S}^{n+\frac{1}{2}}\right)=0, \quad \operatorname{tr}\left(\overline{\mathbf{Q}}^{n+\frac{1}{2}}\right)=0
$$

It then follows from (3.9) that

$$
\frac{1}{\delta t} \operatorname{tr}\left(\mathbf{Q}^{n+1}\right)-\frac{M L}{2} \Delta \operatorname{tr}\left(\mathbf{Q}^{n+1}\right)=0 .
$$

Taking the $L^{2}$ inner product of the above equation with $\operatorname{tr}\left(\mathbf{Q}^{n+1}\right)$, we arrive at

$$
\frac{1}{\delta t}\left\|\operatorname{tr}\left(\mathbf{Q}^{n+1}\right)\right\|^{2}+\frac{M L}{2}\left\|\nabla \operatorname{tr}\left(\mathbf{Q}^{n+1}\right)\right\|^{2}=0,
$$

which implies $\operatorname{tr}\left(\mathbf{Q}^{n+1}\right)=0$. 
In addition, we can show $\mathbf{Q}^{n+1}$ is symmetric. From scheme $(3.2)$, given $\mathbf{Q}^{n}, \mathbf{Q}^{n-1} \in \mathcal{M}$, the transport equations for $\mathbf{Q}_{i j}^{n+1}$ and $\mathbf{Q}_{j i}^{n+1}, i, j=1,2,3$, are the same. Thus, $\mathbf{Q}^{n+1}=\left(\mathbf{Q}^{n+1}\right)^{T}$. Finally we conclude $\mathbf{Q}^{n+1} \in \mathcal{M}_{t r 0}$.

Remark 3.1. For scheme (3.5), we can decouple $\mathbf{u}^{n+1}$ and $p^{n+1}$ in two steps: we solve for $p^{n+1}$ firstly and then $\mathbf{u}^{n+1}$. Taking divergence on both side of (3.5), we obtain a Poisson equation for $p^{n+1}$,

$$
-\Delta p^{n+1}=\frac{2}{\delta t} \nabla \cdot \widetilde{\mathbf{u}}^{n+1}-\Delta p^{n}
$$

The Poisson equation is solved either subject to the Neumann boundary condition $\left.\partial_{\mathbf{n}} p^{n+1}\right|_{\partial \Omega}=0$ or the periodic boundary condition, that is decided by the boundary conditions of other variables. Afterwards, $\mathbf{u}^{n+1}$ is updated by the following formula:

$$
\mathbf{u}^{n+1}=-\frac{\delta t}{2} \nabla\left(p^{n+1}-p^{n}\right)+\widetilde{\mathbf{u}}^{n+1}
$$

Therefore, the scheme defined in (3.2)-(3.5) is equivalent to the following algorithm which is more easily to be implemented.

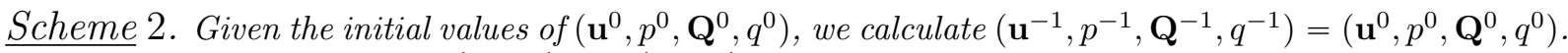
Then, $\forall n \geqslant 0$, we obtain $\left(\mathbf{u}^{n+1}, p^{n+1}, \mathbf{Q}^{n+1}, q^{n+1}\right)$ through the following steps:

Step 1: solve for $\left(\widetilde{\mathbf{u}}^{n+1}, \mathbf{Q}^{n+1}\right)$ from

$$
\left\{\begin{array}{l}
\frac{\widetilde{\mathbf{u}}^{n+1}-\mathbf{u}^{n}}{\delta t}+B\left(\overline{\mathbf{u}}^{n+\frac{1}{2}}, \mathbf{u}_{*}^{n+\frac{1}{2}}\right)=-\nabla p^{n}+\eta \nabla^{2} \mathbf{u}_{*}^{n+\frac{1}{2}}+\nabla \cdot \boldsymbol{\sigma}^{n+\frac{1}{2}}-\widetilde{\mathcal{H}}^{n+\frac{1}{2}} \nabla \overline{\mathbf{Q}}^{n+\frac{1}{2}}, \\
\mathbb{A}\left(\mathbf{Q}^{n+1}, \widetilde{\mathbf{u}}^{n+1}\right)=\mathbb{G}\left(\mathbf{Q}^{n}\right),
\end{array}\right.
$$

where

$$
\widetilde{\mathcal{H}}^{n+\frac{1}{2}}=L \Delta \mathbf{Q}^{n+\frac{1}{2}}-\left(\frac{1}{2} \overline{\boldsymbol{P}}^{n+\frac{1}{2}}:\left(\mathbf{Q}^{n+1}-\mathbf{Q}^{n}\right)+q^{n}\right) \overline{\boldsymbol{P}}^{n+\frac{1}{2}} .
$$

Step 2: solve for $q^{n+1}$ from

$$
q^{n+1}=\overline{\boldsymbol{P}}^{n+\frac{1}{2}}:\left(\mathbf{Q}^{n+1}-\mathbf{Q}^{n}\right)+q^{n} .
$$

Step 3: solve for $p^{n+1}$ from the Poisson equation

$$
-\Delta p^{n+1}=\frac{2}{\delta t} \nabla \cdot \widetilde{\mathbf{u}}^{n+1}-\Delta p^{n}
$$

Step 4: update $\mathbf{u}^{n+1}$ using

$$
\mathbf{u}^{n+1}=-\frac{\delta t}{2} \nabla\left(p^{n+1}-p^{n}\right)+\widetilde{\mathbf{u}}^{n+1}
$$

We next prove the unconditional energy stability of the scheme defined in (3.2)-(3.5) (or its equivalent version in (3.15)-(3.19)) as follows.

Theorem 3.2. The scheme (3.2)-(3.5) (or (3.15)-(3.19)) is unconditionally energy stable, satisfying the discrete energy dissipation law

$$
E^{n+1}-E^{n}=-\delta t\left(\eta\left\|\nabla \mathbf{u}_{*}{ }^{n+\frac{1}{2}}\right\|^{2}+M\left\|\mathcal{H}^{n+\frac{1}{2}}\right\|^{2}\right)
$$

where $E^{n}$ is defined by

$$
E^{n}=\frac{1}{2}\left\|\mathbf{u}^{n}\right\|^{2}+\frac{\delta t^{2}}{8}\left\|\nabla p^{n}\right\|^{2}+\frac{L}{2}\left\|\nabla \mathbf{Q}^{n}\right\|^{2}+\frac{1}{2}\left\|q^{n}\right\|^{2} .
$$


Proof. First, we take the $L^{2}$ inner product of the equation for $\widetilde{\mathbf{u}}^{n+1}, \mathbf{Q}^{n+1}, q^{n+1}$ from (3.2) with $\delta t \mathbf{u}_{*}^{n+\frac{1}{2}}$, $-\delta t \mathcal{H}^{n+\frac{1}{2}}$ and $\delta t q^{n+\frac{1}{2}}$, respectively, to yield

$$
\begin{aligned}
& \frac{1}{2}\left(\left\|\widetilde{\mathbf{u}}^{n+1}\right\|^{2}-\left\|\mathbf{u}^{n}\right\|^{2}\right)+ \delta t\left(\nabla p^{n}, \mathbf{u}_{*}^{n+\frac{1}{2}}\right)+\eta\left\|\nabla \mathbf{u}_{*}^{n+\frac{1}{2}}\right\|^{2} \\
&- \delta t\left(\nabla \cdot \boldsymbol{\sigma}^{n+\frac{1}{2}}, \mathbf{u}_{*}^{n+\frac{1}{2}}\right)+\delta t\left(\mathcal{H}^{n+\frac{1}{2}} \nabla \overline{\mathbf{Q}}^{n+\frac{1}{2}}, \mathbf{u}_{*}^{n+\frac{1}{2}}\right)=0 \\
&-\left(\mathbf{Q}^{n+1}-\mathbf{Q}^{n}, \mathcal{H}^{n+\frac{1}{2}}\right)+ \delta t M\left\|\mathcal{H}^{n+\frac{1}{2}}\right\|^{2} \\
&+\delta t\left(\mathcal{H}^{n+\frac{1}{2}}, \boldsymbol{S}^{n+\frac{1}{2}}\right)-\delta t\left(\mathbf{u}_{*}^{n+\frac{1}{2}} \cdot \nabla \overline{\mathbf{Q}}^{n+\frac{1}{2}}, \mathcal{H}^{n+\frac{1}{2}}\right)=0 \\
& \frac{1}{2}\left(\left\|q^{n+1}\right\|^{2}-\left\|q^{n}\right\|^{2}\right)-\left(q^{n+\frac{1}{2}}\left(\mathbf{Q}^{n+1}-\mathbf{Q}^{n}\right), \overline{\boldsymbol{P}}^{n+\frac{1}{2}}\right)=0 .
\end{aligned}
$$

Using the expression of $\mathcal{H}^{n+\frac{1}{2}}$ in (3.3) and substituting it into (3.23), we derive

$$
\begin{aligned}
\frac{L}{2}\left(\mid \nabla \mathbf{Q}^{n+1}\left\|^{2}-\right\| \nabla \mathbf{Q}^{n} \|^{2}\right) & +\left(\mathbf{Q}^{n+1}-\mathbf{Q}^{n}, q^{n+\frac{1}{2}} \overline{\boldsymbol{P}}^{n+\frac{1}{2}}\right)+\delta t M\left\|\mathcal{H}^{n+\frac{1}{2}}\right\|^{2} \\
& +\delta t\left(\mathcal{H}^{n+\frac{1}{2}}, \boldsymbol{S}^{n+\frac{1}{2}}\right)-\delta t\left(\mathbf{u}_{*}^{n+\frac{1}{2}} \cdot \nabla \overline{\mathbf{Q}}^{n+\frac{1}{2}}, \mathcal{H}^{n+\frac{1}{2}}\right)=0 .
\end{aligned}
$$

Next, taking the $L^{2}$ inner product of (3.5) with $\frac{\delta t}{2}\left(\mathbf{u}^{n+1}+\widetilde{\mathbf{u}}^{n+1}\right)$, we obtain

$$
\frac{1}{2}\left(\left\|\mathbf{u}^{n+1}\right\|^{2}-\left\|\widetilde{\mathbf{u}}^{n+1}\right\|^{2}\right)+\frac{\delta t}{4}\left(\mathbf{u}^{n+1}+\widetilde{\mathbf{u}}^{n+1}, p^{n+1}-p^{n}\right)=0 .
$$

In addition, from (3.5), we obtain the following by taking the $L^{2}$ inner product of (3.5) with $\frac{\delta t^{2}}{2} \nabla p^{n}$ directly,

$$
\frac{\delta t^{2}}{8}\left(\left\|\nabla p^{n+1}\right\|^{2}-\left\|\nabla p^{n}\right\|^{2}-\left\|\nabla\left(p^{n+1}-p^{n}\right)\right\|^{2}\right)-\delta t\left(\nabla p^{n}, \mathbf{u}_{*}^{n+\frac{1}{2}}\right)=0 .
$$

Taking the $L^{2}$ inner product of (3.5) with $\delta t \mathbf{u}^{n+1}$, we have

$$
\frac{1}{2}\left(\left\|\mathbf{u}^{n+1}\right\|^{2}-\left\|\widetilde{\mathbf{u}}^{n+1}\right\|^{2}+\left\|\mathbf{u}^{n+1}-\widetilde{\mathbf{u}}^{n+1}\right\|^{2}\right)=0
$$

From (3.5), we obtain

$$
\frac{\delta t^{2}}{8}\left\|\nabla p^{n+1}-\nabla p^{n}\right\|^{2}=\frac{1}{2}\left\|\mathbf{u}^{n+1}-\widetilde{\mathbf{u}}^{n+1}\right\|^{2} .
$$

Adding equations (3.27), (3.28) and (3.29), we end up with

$$
\frac{1}{2}\left(\left\|\mathbf{u}^{n+1}\right\|^{2}-\left\|\widetilde{\mathbf{u}}^{n+1}\right\|^{2}\right)+\frac{\delta t^{2}}{8}\left(\left\|\nabla p^{n+1}\right\|^{2}-\left\|\nabla p^{n}\right\|^{2}\right)-\delta t\left(\nabla p^{n}, \mathbf{u}_{*}^{n+\frac{1}{2}}\right)=0 .
$$

Similar to Lemma 2.2, we have

$$
\left(\nabla \cdot \boldsymbol{\sigma}^{n+\frac{1}{2}}, \mathbf{u}_{*}^{n+\frac{1}{2}}\right)-\left(\mathcal{H}^{n+\frac{1}{2}}, \boldsymbol{S}^{n+\frac{1}{2}}\right)=0 .
$$

Finally, we combine (3.22), (3.24), (3.25), and (3.30) to obtain

$$
\begin{aligned}
\frac{1}{2}\left\|\mathbf{u}^{n+1}\right\|^{2} & +\frac{\delta t^{2}}{8}\left\|\nabla p^{n+1}\right\|^{2}+\frac{L}{2}\left\|\nabla \mathbf{Q}^{n+1}\right\|^{2}+\frac{1}{2}\left\|q^{n+1}\right\|^{2} \\
& +\delta t \eta\left\|\nabla \mathbf{u}_{*}^{n+\frac{1}{2}}\right\|^{2}+\delta t M\left\|\mathcal{H}^{n+\frac{1}{2}}\right\|^{2}=\frac{1}{2}\left\|\mathbf{u}^{n}\right\|^{2}+\frac{\delta t^{2}}{8}\left\|\nabla p^{n}\right\|^{2}+\frac{L}{2}\left\|\nabla \mathbf{Q}^{n}\right\|^{2}+\frac{1}{2}\left\|q^{n}\right\|^{2} .
\end{aligned}
$$

This concludes the proof.

Remark 3.2. In the Scheme 1 and Scheme 2, the pressure field is decoupled from the other variables through the projection method ( $c f$. the references in the overview of projection method in [15]). One 
alternative scheme to solve the system is using the following "coupled" scheme where the velocity and the pressure are coupled together. The scheme reads as follows.

Given the initial values of $\left(\mathbf{u}^{0}, p^{0}, \mathbf{Q}^{0}, q^{0}\right)$, we set $\left(\mathbf{u}^{-1}, p^{-1}, \mathbf{Q}^{-1}, q^{-1}\right)=\left(\mathbf{u}^{0}, p^{0}, \mathbf{Q}^{0}, q^{0}\right)$. Then $\forall n \geqslant 0$, we obtain $\left(\mathbf{u}^{n+1}, p^{n+1}, \mathbf{Q}^{n+1}, q^{n+1}\right)$

$$
\left\{\begin{array}{l}
\frac{\mathbf{u}^{n+1}-\mathbf{u}^{n}}{\delta t}+B\left(\overline{\mathbf{u}}^{n+\frac{1}{2}}, \mathbf{u}^{n+\frac{1}{2}}\right)=-\nabla p^{n+\frac{1}{2}}+\eta \nabla^{2} \mathbf{u}^{n+\frac{1}{2}}+\nabla \cdot \boldsymbol{\sigma}^{n+\frac{1}{2}}-\mathcal{H}^{n+\frac{1}{2}} \nabla \overline{\mathbf{Q}}^{n+\frac{1}{2}}, \\
\nabla \cdot \mathbf{u}^{n+1}=0 \\
\frac{\mathbf{Q}^{n+1}-\mathbf{Q}^{n}}{\delta t}+\mathbf{u}^{n+\frac{1}{2}} \cdot \nabla \overline{\mathbf{Q}}^{n+\frac{1}{2}}-\boldsymbol{S}^{n+\frac{1}{2}}=M \mathcal{H}^{n+\frac{1}{2}}, \\
q^{n+1}-q^{n}=\overline{\boldsymbol{P}}^{n+\frac{1}{2}}:\left(\mathbf{Q}^{n+1}-\mathbf{Q}^{n}\right),
\end{array}\right.
$$

where

$$
\left\{\begin{array}{l}
\boldsymbol{S}^{n+\frac{1}{2}}=\boldsymbol{S}\left(\nabla \mathbf{u}^{n+\frac{1}{2}}, \overline{\mathbf{Q}}^{n+\frac{1}{2}}\right) \\
\boldsymbol{\sigma}^{n+\frac{1}{2}}=\boldsymbol{\sigma}\left(\overline{\mathbf{Q}}^{n+\frac{1}{2}}, \mathcal{H}^{n+\frac{1}{2}}\right) \\
\overline{\boldsymbol{P}}^{n+\frac{1}{2}}=\boldsymbol{P}\left(\overline{\mathbf{Q}}^{n+\frac{1}{2}}\right) \\
\mathcal{H}^{n+\frac{1}{2}}=L \Delta \mathbf{Q}^{n+\frac{1}{2}}-q^{n+\frac{1}{2}} \overline{\boldsymbol{P}}^{n+\frac{1}{2}}
\end{array}\right.
$$

This scheme is linear and second order in time. This scheme is also suitable for the Stokes flow where the time derivative of $\mathbf{u}$ vanishes. To solve the coupled linear system (in particular velocity field $\mathbf{u}$ and pressure $p$ ), we need an efficient preconditioner (cf. [14]). Moreover, we can prove that the coupled scheme still follows the energy law with a similar proof as Scheme 1. We omit the details here due to the limit of space.

Theorem 3.3. The scheme defined in (3.2)-(3.5) (or (3.15)-(3.19)) admits a unique solution.

Proof. Assuming that $\widetilde{\mathbf{u}}^{1}, \mathbf{Q}^{1}, q^{1}$ and $\widetilde{\mathbf{u}}^{2}, \mathbf{Q}^{2}, q^{2}$ are two solutions of (3.2), we let

$$
\left\{\begin{array}{l}
\widetilde{\mathbf{u}}^{0}=\widetilde{\mathbf{u}}^{1}-\widetilde{\mathbf{u}}^{2}, \\
\mathbf{Q}^{0}=\mathbf{Q}^{1}-\mathbf{Q}^{2}, \\
q^{0}=q^{1}-q^{2} .
\end{array}\right.
$$

Thus $\widetilde{\mathbf{u}}^{0}, \mathbf{Q}^{0}, q^{0}$ are the solutions of the following system

$$
\begin{aligned}
& \frac{1}{\delta t} \widetilde{\mathbf{u}}^{0}+B\left(\overline{\mathbf{u}}^{n+\frac{1}{2}}, \frac{\widetilde{\mathbf{u}}^{0}}{2}\right)=\eta \Delta \frac{\widetilde{\mathbf{u}}^{0}}{2}+\nabla \cdot \boldsymbol{\sigma}\left(\overline{\mathbf{Q}}^{n+\frac{1}{2}}, \frac{\mathcal{H}_{1}^{n+1}-\mathcal{H}_{2}^{n+1}}{2}\right) \\
& -\frac{\mathcal{H}_{1}^{n+1}-\mathcal{H}_{2}^{n+1}}{2} \nabla \overline{\mathbf{Q}}^{n+\frac{1}{2}} \\
& \frac{\mathbf{Q}^{0}}{\delta t}+\frac{\widetilde{\mathbf{u}}_{0}}{2} \nabla \overline{\mathbf{Q}}^{n+\frac{1}{2}}-\boldsymbol{S}\left(\frac{\nabla \widetilde{\mathbf{u}}^{0}}{2}, \overline{\mathbf{Q}}^{n+\frac{1}{2}}\right)=M \frac{\mathcal{H}_{1}^{n+1}-\mathcal{H}_{2}^{n+1}}{2}, \\
& q^{0}=\overline{\boldsymbol{P}}^{n+\frac{1}{2}}: \mathbf{Q}^{0} .
\end{aligned}
$$

By taking the $L^{2}$ inner product of (3.36) with $\widetilde{\mathbf{u}}^{0},(3.37)$ with $-\left(\mathcal{H}_{1}^{n+1}-\mathcal{H}_{2}^{n+1}\right)$, (3.38) with $q_{0}$, and combining all equalities, we arrive at

$$
\frac{1}{\delta t}\left\|\widetilde{\mathbf{u}}^{0}\right\|^{2}+\frac{L}{\delta t}\left\|\nabla \mathbf{Q}^{0}\right\|^{2}+\left\|q^{0}\right\|^{2}+\frac{M}{2}\left\|\left(\mathcal{H}_{1}^{n+1}-\mathcal{H}_{2}^{n+1}\right)\right\|^{2}=0 .
$$

We deduce that $\tilde{\mathbf{u}}^{0}=0, q^{0}=0$, and $\mathbf{Q}^{0}=0$. Since the uniqueness and existence for (3.5) is easy to establish, we complete the proof. 
3.2. Scheme for the Q-tensor model. If hydrodynamic effects are so weak that they can be neglected, model (2.14) reduces to a nematodynamic equation system,

$$
\left\{\begin{aligned}
\mathbf{Q}_{t} & =M(L \Delta \mathbf{Q}+q \boldsymbol{P}(\mathbf{Q})) \\
q_{t} & =\boldsymbol{P}(\mathbf{Q}): \mathbf{Q}_{t}
\end{aligned}\right.
$$

We refer to it as the reduced model in this paper. Following the same strategy in the development of schemes for the coupled model, we devise a linear, second order numerical scheme as follows:

\section{Scheme 3 .}

$$
\left\{\begin{array}{l}
\frac{\mathbf{Q}^{n+1}-\mathbf{Q}^{n}}{\delta t}=M\left(L \Delta \mathbf{Q}^{n+\frac{1}{2}}-q^{n+\frac{1}{2}} \overline{\boldsymbol{P}}^{n+\frac{1}{2}}\right), \\
q^{n+1}-q^{n}=\overline{\boldsymbol{P}}^{n+\frac{1}{2}}:\left(\mathbf{Q}^{n+1}-\mathbf{Q}^{n}\right) .
\end{array}\right.
$$

Applying identity (3.6), we write $\mathbf{Q}^{n+1}$ as the solution of the following linear system

$$
\widehat{\mathbb{A}}\left(\mathbf{Q}^{n+1}\right)=\mathbb{G}\left(\mathbf{Q}^{n}\right)
$$

where

$$
\widehat{\mathbb{A}}\left(\mathbf{Q}^{n+1}\right)=\frac{1}{\delta t} \mathbf{Q}^{n+1}-\frac{M L}{2} \Delta \mathbf{Q}^{n+1}+\frac{M}{2}\left(\overline{\boldsymbol{P}}^{n+\frac{1}{2}}: \mathbf{Q}^{n+1}\right) \overline{\boldsymbol{P}}^{n+\frac{1}{2}}
$$

We have the following result for the linear operator $\widehat{\mathbb{A}}$.

Theorem 3.4. The linear operator $\widehat{\mathbb{A}}: \mathcal{M}_{t r 0} \rightarrow \mathcal{M}_{t r 0}$ is a positive definite operator, thus the linear system (3.41) admits a unique solution.

Proof. For any $\mathbf{Q} \in \mathcal{M}_{\text {tr } 0}$, we have $\widehat{\mathbb{A}} \mathbf{Q} \in \mathcal{M}_{\text {tr } 0}$ from Theorem 3.1.

For any $\left(\mathbf{Q}_{1}, \mathbf{Q}_{2}\right) \in \mathcal{M}_{t r 0}$ satisfying the boundary condition (1.12),

$$
\begin{aligned}
\left(\widehat{\mathbb{A}} \mathbf{Q}_{1}, \mathbf{Q}_{2}\right) & =\frac{1}{\delta t}\left(\mathbf{Q}_{1}, \mathbf{Q}_{2}\right)+\frac{M}{2}\left(\overline{\boldsymbol{P}}^{n+\frac{1}{2}}: \mathbf{Q}_{1}, \overline{\boldsymbol{P}}^{n+\frac{1}{2}}: \mathbf{Q}_{2}\right)+\frac{M L}{2}\left(\nabla \mathbf{Q}_{1}, \nabla \mathbf{Q}_{2}\right) \\
& =\left(\mathbf{Q}_{1}, \widehat{\mathbb{A}} \mathbf{Q}_{2}\right),
\end{aligned}
$$

Thus the linear operator $\widehat{\mathbb{A}}$ is self-adjoint.

Moreover, for any $\mathbf{Q} \in \mathcal{M}_{t r 0}$, one can easily derive

$$
(\widehat{\mathbb{A}} \mathbf{Q}, \mathbf{Q})=\frac{1}{\delta t}\|\mathbf{Q}\|^{2}+\frac{1}{2}\left\|\overline{\boldsymbol{P}}^{n+\frac{1}{2}}: \mathbf{Q}\right\|^{2}+\frac{M L}{2}\|\nabla \mathbf{Q}\|^{2} \geqslant 0 .
$$

The "=" is valid if and only if $\mathbf{Q}=\mathbf{0}$. Then, the proof is complete.

Remark 3.3. The unconditional energy stability of scheme (3.41) for the simple model (3.40) can be established similar to Theorem 3.2. We thus omit the details here. We emphasize that scheme (3.42) is extremely efficient since the linear operator in (3.42) is positive definite, thus it can be solved by many efficient linear solvers, e.g., BiCG, GMRES, or other Krylov subspace methods.

Remark 3.4. Although we consider only time discrete schemes in this paper, the results here an be carried over to any consistent finite-dimensional Galerkin type approximations since the proofs are all based on a variational formulation with all test functions in the same space as the space of the trial functions. About the standard analysis technique when the space discretization is involved, we refer to [31, 32].

\section{Numerical Results}

We now present numerical experiments in two dimensions to validate the theoretical results derived in the previous section and demonstrate the efficiency, energy stability and accuracy of the proposed numerical schemes. Here, the semi-discrete scheme 1 is further discretized in space via the equal-spaced, 


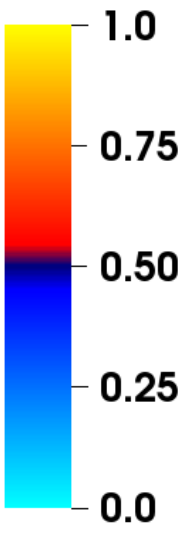

(a) Color bar

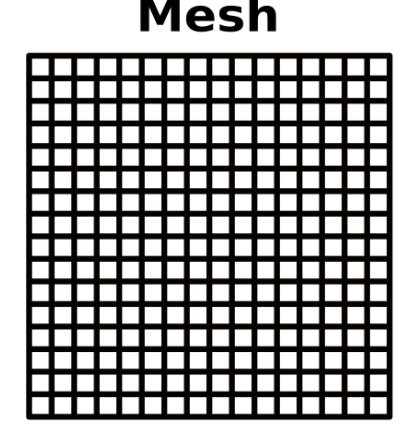

Block
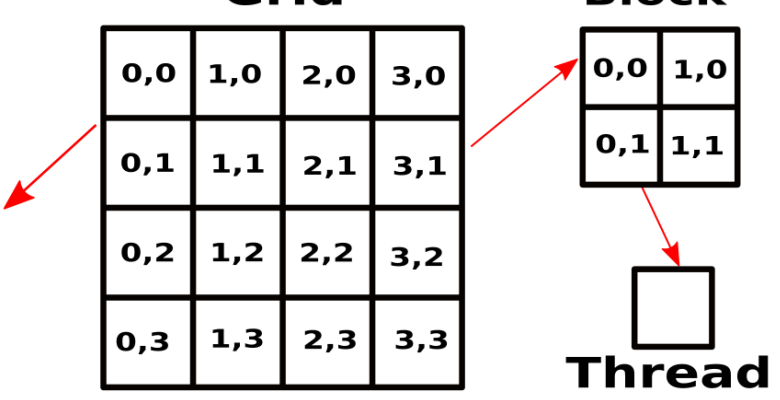

FiguRE 1. Some schematics. (a) the color bar used in this paper; (b) mesh mapping into GPU grids.

finite difference method. Then, it is implemented on a GPU for computational efficiency, as each mesh point could be mapped into a single computational thread, shown in Figure 1(b).

In the following, we will use the color map depicted in Figure 1(a) to denote changes of a scalar and use the following model parameter values (unless otherwise stated):

$$
a=-0.2, b=1, c=1, L=1.0 \times 10^{-3}, A_{0}=500, M=1 .
$$

We note that the choice of the gauge value $A_{0}$ in the free energy functional does not affect the results at all. Thus, its value can be chosen as any value so long as it serves our purposes, i.e. the definition of $q(\mathbf{Q})$ makes sense.

4.1. Accuracy and stability test. We first test convergence rates of Scheme 1. The following initial conditions

$$
\mathbf{Q}_{0}=\mathbf{n}_{0} \mathbf{n}_{0}^{T}-\frac{\left\|\mathbf{n}_{0}\right\|^{2}}{3} \mathbf{I}, \quad \mathbf{n}_{0}=(\cos (2 \pi y) \cos (2 \pi x), \cos (2 \pi y) \cos (2 \pi x), 0)
$$

are used. We perform the refinement test of the time step size. The numerical errors are calculated as the difference between the solution of coarse time step and that of the adjacent finer time step. We present the Cauchy sequence of $L^{2}$ errors at $t=1$ with different time step sizes in Table 4.1 . We observe that the scheme is second order accurate.

4.1.1. Stability test. We further compare the stability of the Scheme 1 with the second-order implicit scheme developed in [47]. We choose $L_{x}=L_{y}=2$, and $a=-\frac{1}{3}, b=-4, c=4$. The initial condition is set as follows,

$$
\mathbf{Q}_{0}=\frac{\mathbf{n}_{0} \mathbf{n}_{0}^{T}}{\left\|\mathbf{n}_{0}\right\|^{2}}-\frac{1}{3} \mathbf{I}, \quad \mathbf{n}_{0}=(\cos (k \theta), \sin (k \theta), 0), \quad \theta=\operatorname{atan} 2\left(x-0.5 L_{x}, y-0.5 L_{y}\right),
$$

where $\operatorname{atan} 2(x, y)$ is the standard function to calculates the arctangent of $y / x$, and $k=4$. In Table 4.2, we show the free energy at $t=1$ calculated with different time steps using both schemes. We notice that Scheme 1 converges to the correct solution, as the time step decreases. But the implicit scheme of [47] does not converge when using relatively larger time steps. That means scheme 1 can allow much larger time step from the stability concern than the nonlinear implicit scheme of [47]. On the other hand, due 


\begin{tabular}{|c|c|c|c|c|c|c|}
\hline$\delta t$ & $u$ & Order & $v$ & Order & $q$ & Order \\
\hline 0.01 & $4.84 \mathrm{e}-2$ & & $4.84 \mathrm{e}-2$ & & $4.39 \mathrm{e}-4$ & \\
\hline 0.005 & $3.07 \mathrm{e}-3$ & 3.98 & $3.07 \mathrm{e}-3$ & 3.98 & $1.18 \mathrm{e}-4$ & 1.90 \\
\hline 0.0025 & $1.25 \mathrm{e}-4$ & 4.59 & $1.27 \mathrm{e}-3$ & 4.62 & $3.22 \mathrm{e}-5$ & 1.87 \\
\hline 0.00125 & $3.34 \mathrm{e}-5$ & 1.89 & $3.44 \mathrm{e}-5$ & 1.91 & $8.65 \mathrm{e}-6$ & 1.90 \\
\hline 0.000625 & $8.75 \mathrm{e}-6$ & 1.93 & $9.05 \mathrm{e}-6$ & 1.93 & $2.27 \mathrm{e}-6$ & 1.93 \\
\hline 0.0003125 & $2.22 \mathrm{e}-6$ & 1.98 & $2.30 \mathrm{e}-6$ & 1.98 & $5.94 \mathrm{e}-7$ & 1.94 \\
\hline$\delta t$ & $Q_{11}$ & Order & $Q_{12}$ & Order & $Q_{22}$ & Order \\
\hline 0.01 & $7.48 \mathrm{e}-2$ & & $1.53 \mathrm{e}-2$ & & $7.53 \mathrm{e}-2$ & \\
\hline 0.005 & $3.85 \mathrm{e}-2$ & 0.96 & $8.11 \mathrm{e}-2$ & 0.92 & $3.92 \mathrm{e}-2$ & 0.94 \\
\hline 0.0025 & $8.91 \mathrm{e}-3$ & 2.11 & $2.54 \mathrm{e}-2$ & 1.67 & $8.91 \mathrm{e}-3$ & 2.14 \\
\hline 0.00125 & $2.46 \mathrm{e}-3$ & 1.85 & $9.13 \mathrm{e}-4$ & 1.48 & $2.46 \mathrm{e}-3$ & 1.89 \\
\hline 0.000625 & $6.48 \mathrm{e}-4$ & 1.93 & $2.68 \mathrm{e}-4$ & 1.77 & $6.46 \mathrm{e}-4$ & 1.92 \\
\hline 0.0003125 & $1.65 \mathrm{e}-4$ & 1.97 & $7.13 \mathrm{e}-5$ & 1.91 & $1.64 \mathrm{e}-4$ & 1.97 \\
\hline
\end{tabular}

TABLE 4.1. Cauchy convergence test for Scheme 1. The errors for velocity $\mathbf{u}=(u, v), q$ and $\mathbf{Q}$ are measured in the $L^{2}$ norm at $t=0.5$, with $\delta t=\frac{0.01}{2^{k}}, k=0,1, \cdots, 6$ and the default model parameter values. The numerical errors are calculated as the difference between the solution of coarse time step and that of the adjacent finer time step.

\begin{tabular}{c|c|c|c|c|c|c}
\hline$\delta t$ & 0.02 & 0.01 & 0.005 & 0.0025 & 0.00125 & $1.0 \mathrm{e}-4$ \\
\hline \hline Energy & -0.2546 & -0.2494 & -0.2479 & -0.2475 & -0.2475 & -0.2475 \\
\hline Energy & $\times$ & $\times$ & $\times$ & $\times$ & -0.2475 & -0.2475 \\
\hline
\end{tabular}

TABLE 4.2. The stability comparison of the Scheme 1 with the second-order nonlinear implicit scheme in [47]. The energy at $t=1$ are shown, where the first row of energy is calculated by scheme 1 and the second row of energy is calculated by the second-order nonlinear implicit scheme in [47]. The " $\times$ " sign indicates the numerical solver does not converge.

to the second order accuracy, Scheme 1 can provide acceptable results even with very large time step. For instance, when $\delta t=0.01$, the relative error is only $0.77 \%$.

4.2. Dynamics of defects in liquid crystals. In this subsection, we study defect dynamics in flows of liquid crystals numerically using the implemented linear scheme. We conduct a study of a point +1 defect subject to the Dirichlet boundary condition on $\mathbf{Q}$. This study aims to demonstrate the initial point +1 
defect (initial data) is unstable, and how the degree 1 defect splits into two $1 / 2$ defects and then evolves to reach a steady state. Specifically, we use the default parameter values and a domain $\left[0, L_{x}\right] \times\left[0, L_{y}\right]$ in 2-dimensional space, where $L_{x}=L_{y}=2$. We use the no-slip boundary condition for $\mathbf{u},\left.\mathbf{u}\right|_{\partial \Omega}=0$ and a Dirichlet boundary condition for $\mathbf{Q}$,

$$
\left.\mathbf{Q}\right|_{\partial \Omega}=\frac{\mathbf{n}_{0} \mathbf{n}_{0}^{T}}{\left\|\mathbf{n}_{0}\right\|^{2}}-\frac{1}{3} \mathbf{I}, \quad \mathbf{n}_{0}=\left(x-0.5 L_{x}, y-0.5 L_{y}, 0\right)^{T} .
$$

For the example shown in Figure 2, the initial value of $\mathbf{Q}_{\mathbf{0}}$ is given by

$$
\mathbf{Q}_{0}=\frac{\widehat{\mathbf{n}}_{0} \widehat{\mathbf{n}}_{0}^{T}}{\left\|\widehat{\mathbf{n}}_{0}\right\|^{2}}-\frac{1}{3} \mathbf{I}, \widehat{\mathbf{n}}_{0}=\left(x-0.25 L_{x}, y-0.25 L_{y}, 0\right)^{T} .
$$

We compute the planar mode where $Q_{x z}, Q_{y z}=0$ initially. We note that these two components remain zero for all time. This means that $\mathbf{e}=(0,0,1)^{T}$ is always an eigenvector of $\mathbf{Q}$ and $Q_{z z}$ is the corresponding eigenvalue. In the entire simulation, the eigenvalue is not the largest. So, the major director, the eigenvector corresponding to the largest eigenvalue of $\mathbf{Q}$ is on the $x y$ plane. The liquid crystal orientation on the $x y$ plane is primarily described by the major director and the eigenvalue difference of the two eigenvalues corresponding to the two eigenvectors on the $x y$ plane, which are plotted in Figure 2 . The velocity field and the energy density function with respect to time are also shown. The eigenvalue difference of the two eigenvalues corresponding to the eigenvectors on the $x y$ plane is a true measure of the degree of orientation of the liquid crystal. When the difference is zero, it represents a state of the liquid crystal whose orientation on the $x y$ plane is isotropic and dominant. We refer to this state as the defect, which may include an isotropic state or an oblate state. From the simulation in Figure 2, we observe that the initially imposed +1 defect is not stable so that it splits into two $+\frac{1}{2}$ defects over time subject to the Dirichlet boundary condition. The two $\frac{1}{2}$ defects move away from each other slowly inducing a weak velocity field shown in Figure 2(m-n), where two pairs of vortices are shown existing around the defects when they are near the boundary. One pair is much weaker than the other. When the defect pair moves away from the boundary, the weaker vortex pair goes away leaving the stronger vortex pair correlating with the pair of defect.

4.3. Effect of hydrodynamics on liquid crystal dynamics. In this example, we examine how hydrodynamics affect liquid crystal dynamics by numerically integrating both the hydrodynamic model (2.14) and the nematodynamic model (3.40) and then study them comparatively. We use the same initial data, the set of parameter values and boundary conditions whenever appropriate. Namely, we use the default parameter values together with $\eta=0.1$, and $\lambda=1$ in this example. The initial data are given by

$$
\mathbf{Q}_{0}=\frac{\mathbf{n}_{0} \mathbf{n}_{0}^{T}}{\left\|\mathbf{n}_{0}\right\|^{2}}-\frac{1}{3} \mathbf{I}, \quad \mathbf{n}_{0}= \begin{cases}(1,0,0)^{T}, & \sqrt{(x-1)^{2}+(y-1)^{2}}<0.4, \\ (0,1,0)^{T}, & \text { otherwise, }\end{cases}
$$

and the boundary conditions are given by

$$
\left.\mathbf{Q}\right|_{\partial \Omega}=\frac{\widehat{\mathbf{n}}_{0} \widehat{\mathbf{n}}_{0}^{T}}{\left\|\widehat{\mathbf{n}}_{0}\right\|^{2}}-\frac{1}{3} \mathbf{I}, \widehat{\mathbf{n}}_{0}=(0,1,0)^{T},\left.\quad \mathbf{u}\right|_{\partial \Omega}=0 .
$$

There exists a mismatch in orientation in the circular region and the rest of the domain initially. We will see how this mismatch amplifies over time.

The simulation with hydrodynamics is shown in Figure 3 while the one without hydrodynamics is given in Figure 4. One can easily see that dynamics predicted by the two models are totally different. The defects in the simulation of the hydrodynamic model consist of two pairs of $\pm \frac{1}{2}$ defects that eventually annihilate each other. Whereas, in the model without hydrodynamics, the defects stay in a ring form and the ring shrinks with time. Eventually, the ring disappears. This simulation shows hydrodynamics has a significant impact on transient dynamics of liquid crystals and nematodynamics alone would not render the correct defect dynamics. Therefore, any conclusions drawn from the nematodynamics need to 


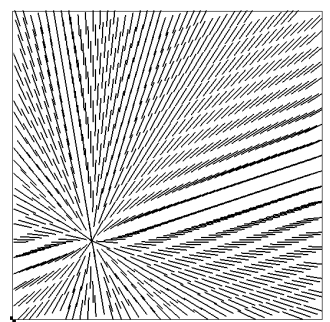

(a)

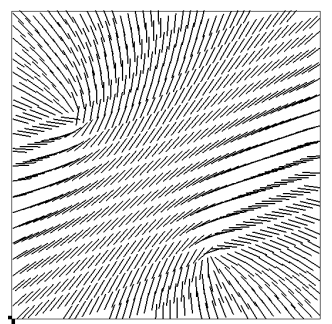

(e)

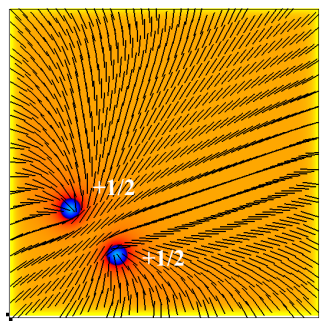

(i)

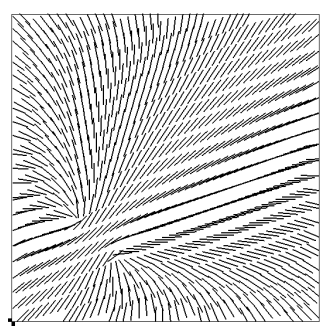

(b)

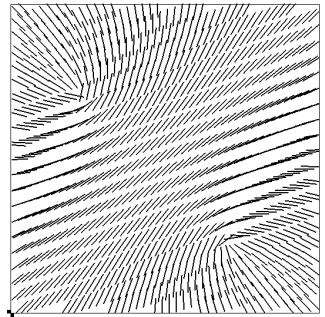

(f)

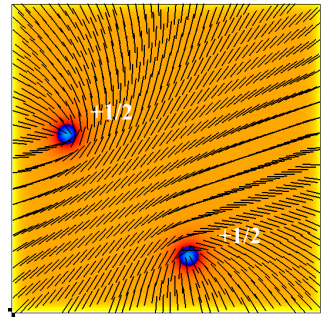

(j)

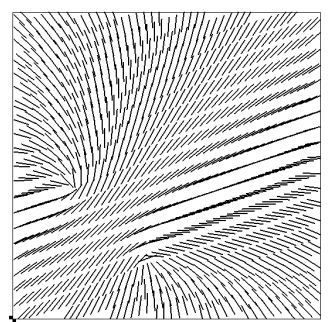

(c)

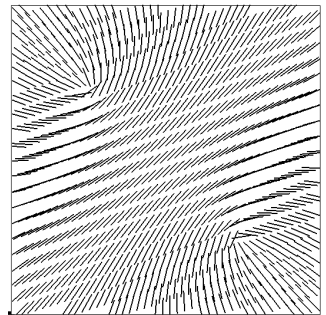

(g)

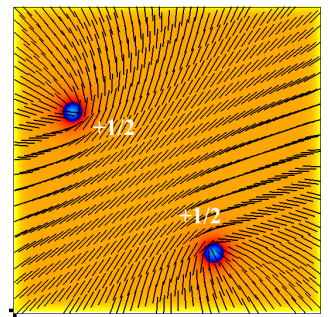

(k)

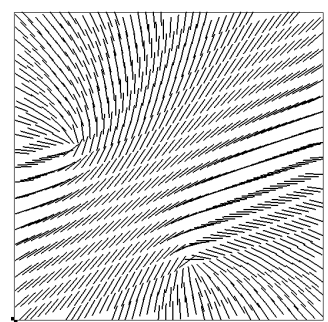

(d)

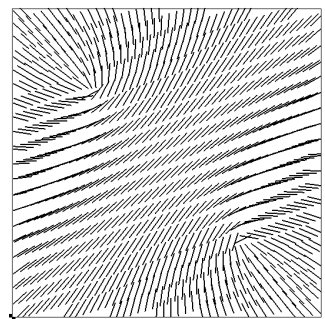

(h)

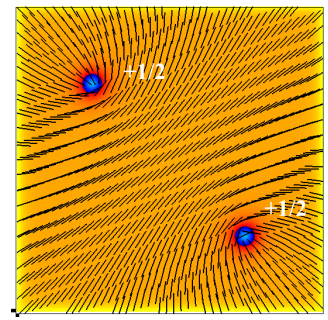

(1)

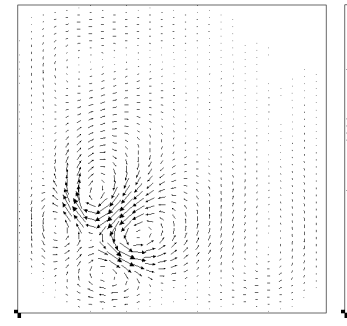

(m)

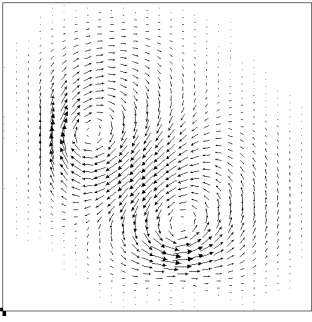

(n)

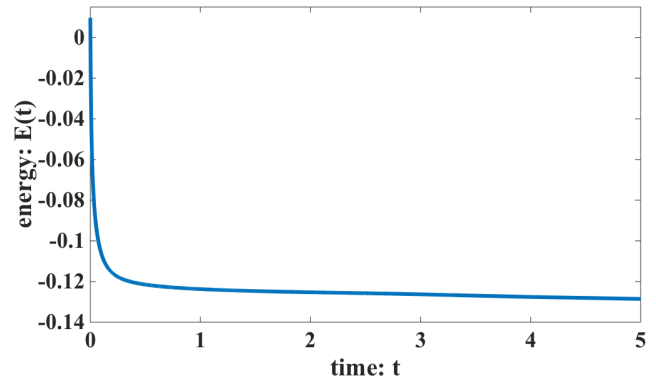

(o)

Figure 2. Defect dynamics. This figure shows that an initially unstable +1 defect splits into two $+\frac{1}{2}$ defects and the two defects remain stable subject to the Dirichlet boundary condition. (a-h) The liquid crystal director orientation on the $x y$ plane at $t=$ $0,5,10,25,50,100,200,250$, respectively. (i-l) The color map of the eigenvalue difference between the two nonzero eigenvalues for $\mathbf{Q}+\frac{1}{3} \mathbf{I}$ on the xy plane at time $t=5,25,50,250$, respectively. The color bar is given in Figure 1. (m-n) The velocity field at $t=5,25$ with the maximum value $1.043 \times 10^{-3}$ and $2.991 \times 10^{-3}$, respectively. (o) The energy as a function of time, which approaches a constant asymptotically in time. 
be scrutinized. We remark that the knee or step in the energy plot in Figure 3(n) and Figure 4(n) is due to the annihilation of defects, at which energy decreases slightly.

4.4. Anchoring effect at the boundary. In this example, we examine how anchoring boundary conditions influence liquid crystal dynamics. We use the same initial values, and the set of parameter values, but different boundary conditions. The domain is set at $L_{x}=2, L_{y}=1$. In the simulation shown in Figure 5, we impose the Dirichlet boundary condition for $\mathbf{Q}$ :

$$
\left.\mathbf{Q}\right|_{\partial \Omega}=\frac{\mathbf{n}_{0} \mathbf{n}_{0}^{T}}{\left\|\mathbf{p}_{0}\right\|^{2}}-\frac{1}{3} \mathbf{I}, \quad \mathbf{n}_{0}=\left(x-0.5 L_{x}, y-0.5 L_{y}, 0\right)^{T} .
$$

In the simulation shown in Figure 6, we employ the Neumann boundary condition for Q:

$$
\frac{\partial \mathbf{Q}}{\partial \mathbf{n}}=0
$$

By comparison, we observe a huge difference in terms of defect dynamics with the two different boundary conditions, indicating the dominating role played by the boundary anchoring condition in defect dynamics in liquid crystals. With the Dirichlet boundary condition, we observe a large number of defect creation and annihilation. Whereas the number of defects are considerably less in the case with the Neumann boundary condition. In the case of the Dirichlet boundary condition, there are four $\frac{1}{2}$ and two $-\frac{1}{2}$ defects along the boundary and one cluster (4) of $-\frac{1}{2}$ defects in the middle near the left and another cluster (4) of $\frac{1}{2}$ defect in the middle near the right. The defects in the clusters expel from each other. Each of the four $-\frac{1}{2}$ defects in the left cluster is attracted to the nearest $\frac{1}{2}$ defect. So are the $\frac{1}{2}$ defects in the right cluster. Defect annihilation takes places among many pairs of defects. At the end of the simulation, only two defects far away from each other survive, both of which are $\frac{1}{2}$ defects. In (a), the total defect count is eight $\frac{1}{2}$ defects and six $-\frac{1}{2}$ defects.

In the case of Neumann boundary conditions, only two clusters of defects exist, each of which is composed of four $\frac{1}{2}$ defects. The left cluster consists of all negative ones while the right cluster is composed of all positive ones. The pair of defects in the middle attract each other while the other six close to the boundary are attracted to the boundary. The middle pair annihilate each other, whereas the others all moved out of the domain. At the end of the simulation, all defects disappear from the computational domain.

This comparison indicates that the defects in the case of Dirichlet boundary condition tend to annihilate each other so that the number of surviving defects is the difference between the positive and negative defects initially, whereas, the defects in the Neumann boundary condition tend to migrate out of the domain after some near-neighbor annihilation. In the end, no defect survives in the computational domain. Hence, the boundary condition is instrumental to long time dynamics of defects in the liquid crystal system.

4.5. Defect dynamics in a sheared cavity. Finally, we consider a cavity flow of liquid crystals subject to a boundary shear in the domain $\left[0, L_{x}\right] \times\left[0, L_{y}\right], L_{x}=L_{y}=2$, where the boundary condition is set as

$$
\mathbf{u}_{x=0}=\mathbf{u}_{x=L_{x}}=\mathbf{u}_{y=0}=0, \quad \mathbf{u}_{y=L_{y}}=1,\left.\quad \frac{\partial \mathbf{Q}}{\partial \mathbf{n}}\right|_{\partial \Omega}=0 .
$$

The numerical simulations are summarized in Figure 7, 8, respectively. We see that defects shed off near the shearing boundary due to shear. As time goes by, more defects are created by shear within the cavity flow geometry. The orientational field, represented by the principal eigenvector or major director, at various time slot is shown in Figure 7, where the flow patterns become increasingly heterogeneous in space with time. Figure 8 depicts the director orientation together with the difference of eigenvalues of $\mathbf{Q}$ corresponding to the two eigenvectors on the xy plane. Corresponding to the increasingly complex flow pattern, the orientational structure also becomes more complex and heterogeneous. 


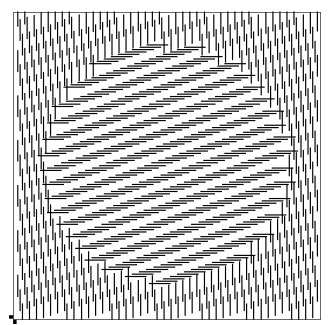

(a)

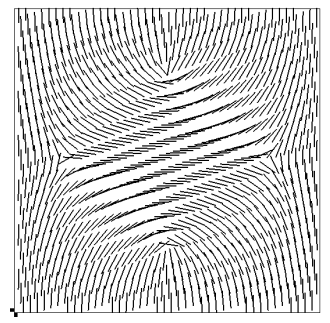

(e)

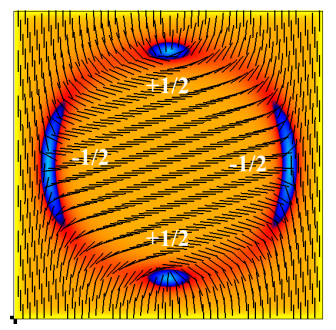

(i)

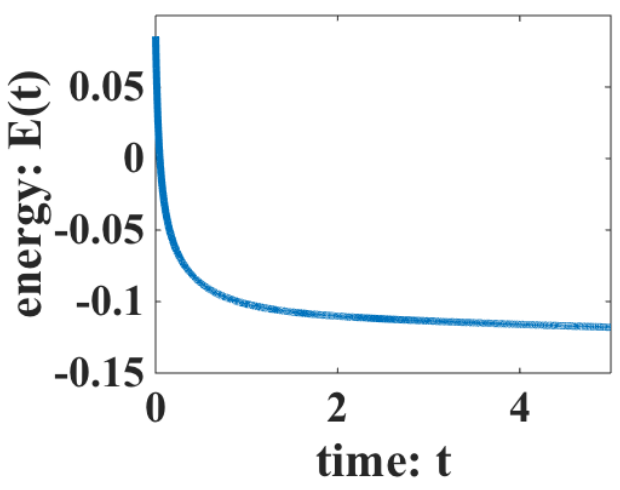

(m) Zoomed Energy

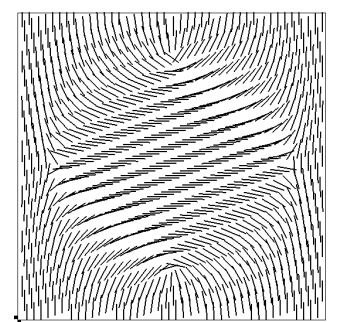

(c)

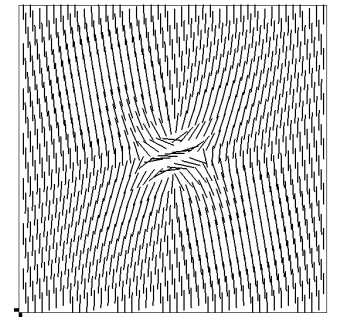

(g)

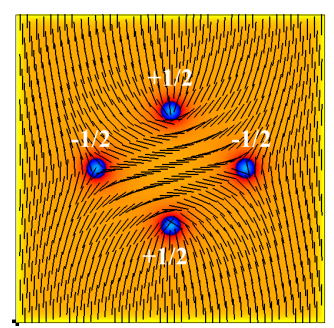

(k)

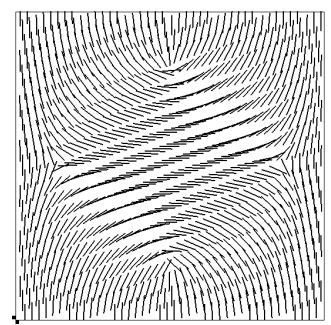

(d)

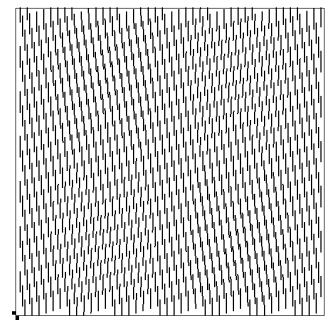

(h)

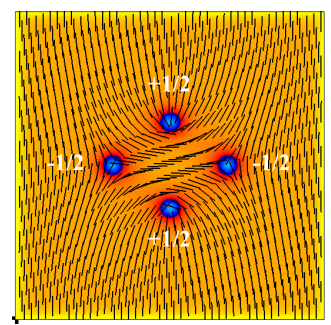

(1)

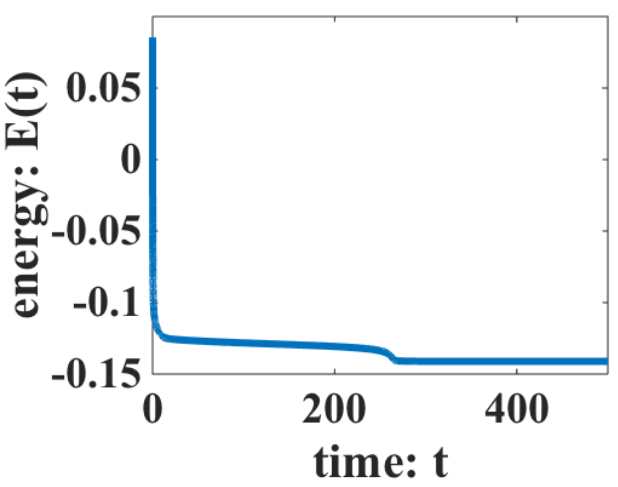

(n) Energy

FIGURE 3. Dynamics of defects with hydrodynamics. (a-h) liquid crystal major director orientation on the $x y$ plane at time $t=0,5,10,25,50,125,250,300$, respectively. (i-1) the difference of eigenvalues on the $x y$ plane for $\mathbf{Q}+\frac{1}{3} \mathbf{I}$ at time $t=5,100,150,200$, respectively. (m)The zoomed view of the energy plot initially. (n) The energy plot over the entire time period of simulation. 


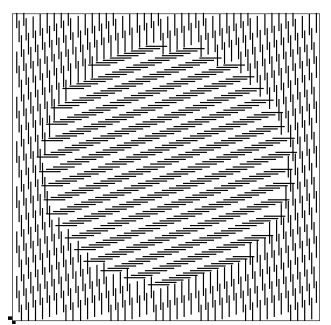

(a)

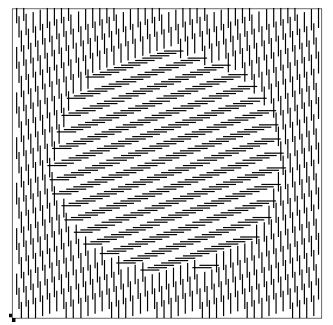

(e)

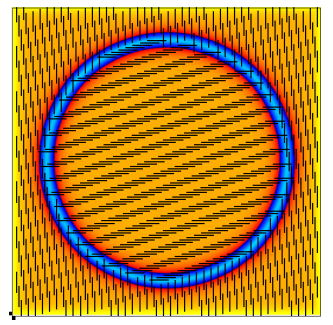

(i)

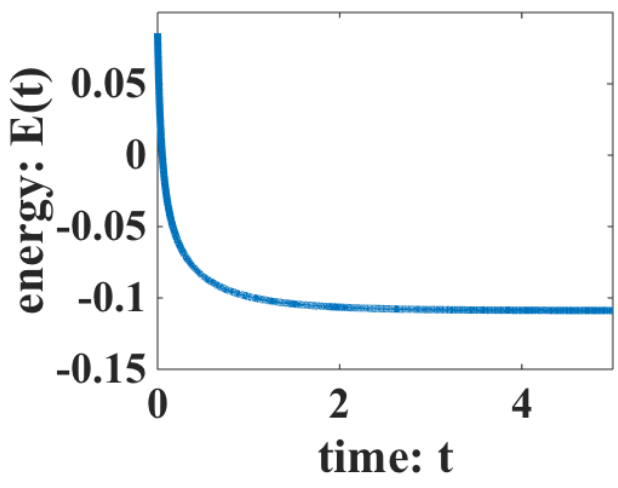

(m) Zoomed Energy

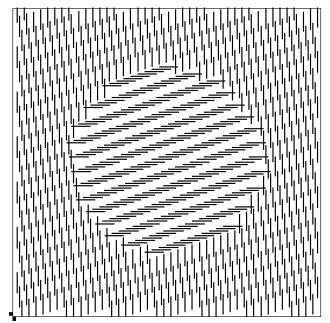

(f)

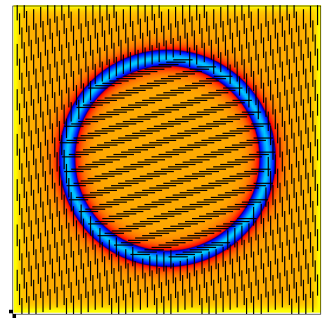

(j)

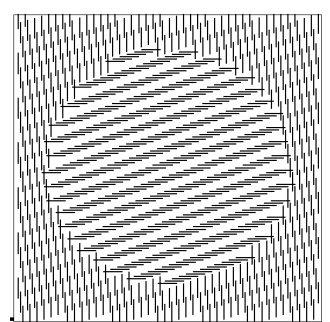

(c)

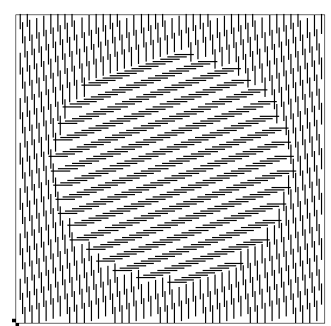

(d)

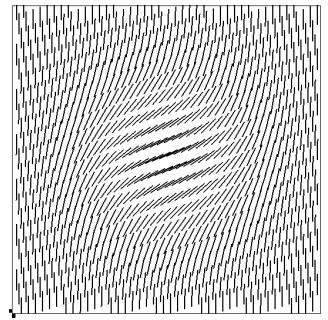

(g)

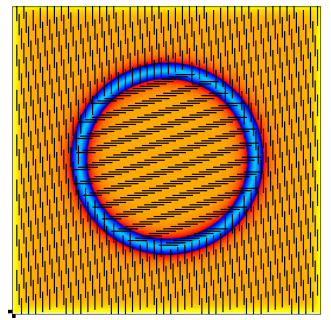

$(\mathrm{k})$

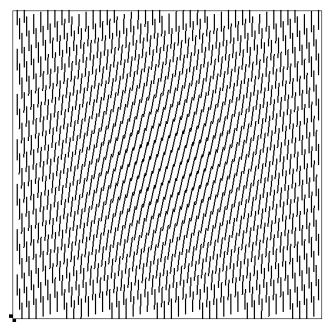

(h)

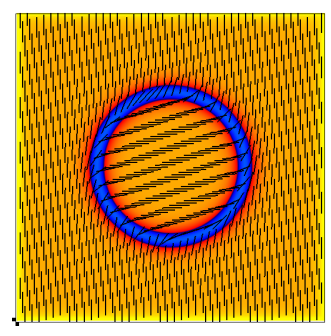

(l)

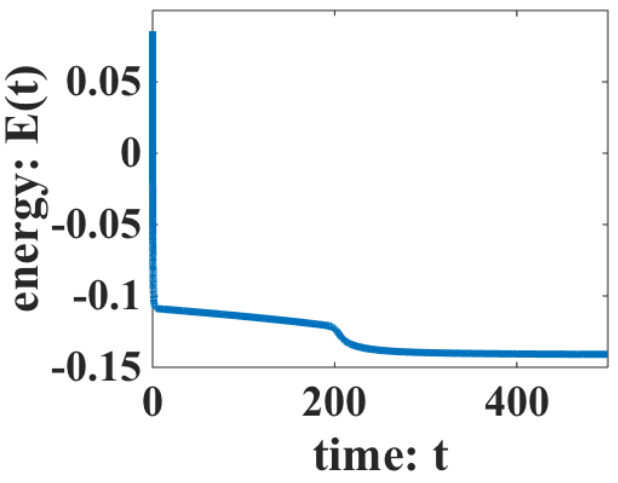

(n) Energy

Figure 4. Dynamics of defects without hydrodynamics-nematodynamics. (a-h) The liquid crystal major director orientation in the $x y$ plane at time $t=$ $0,5,10,25,50,125,250,500$, respectively. (i-l) The difference of eigenvalues on the $x y$ plane for $\mathbf{Q}+\frac{1}{3} \mathbf{I}$ at time $t=5,100,150,200$, respectively. (m) The zoomed view of the energy plot initially. (n) The energy plot for the entire period of simulation. 


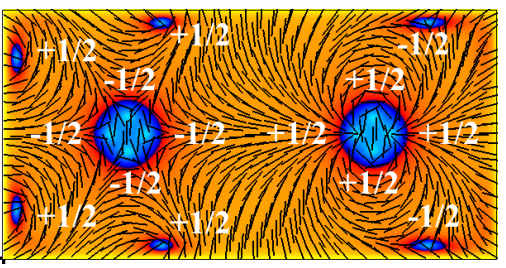

(a)

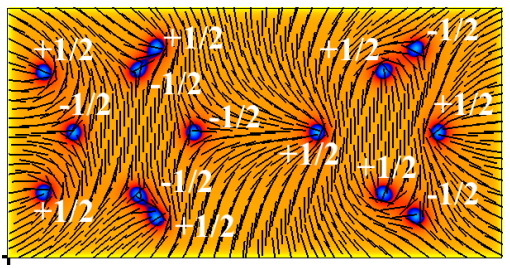

(d)

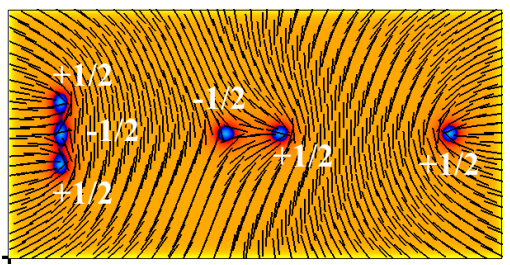

(g)

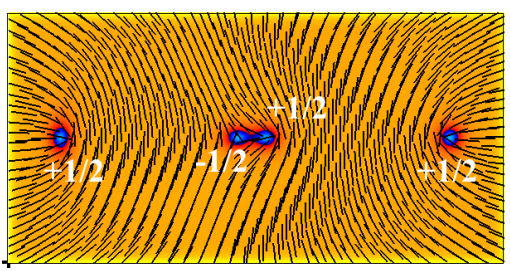

(j)

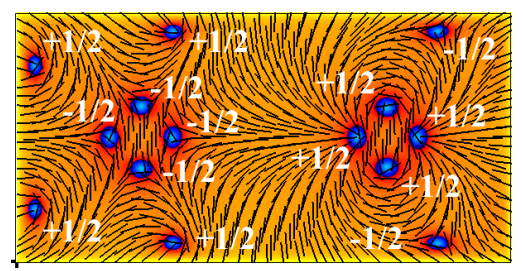

(b)

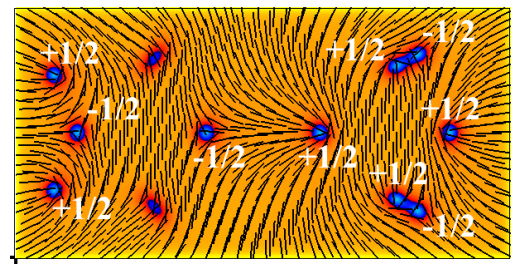

(e)

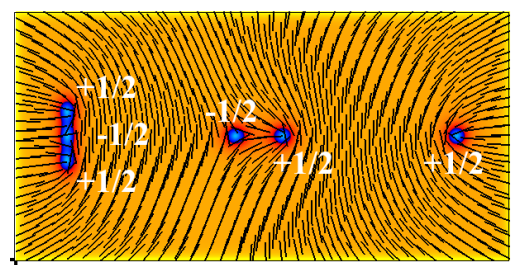

(h)

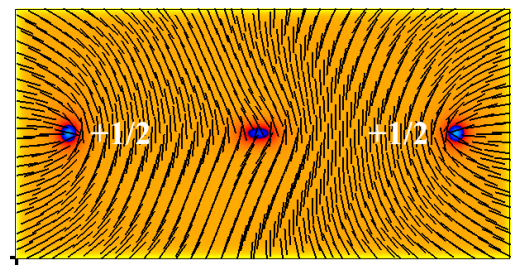

(k)

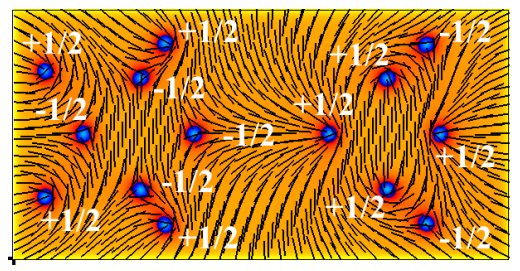

(c)

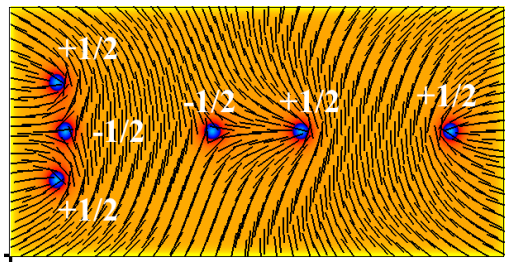

(f)

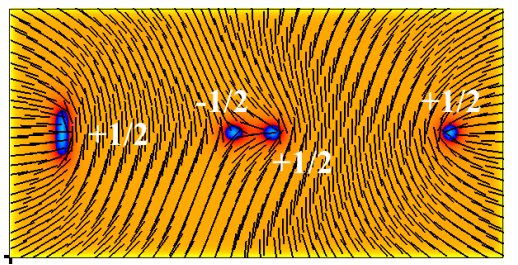

(i)

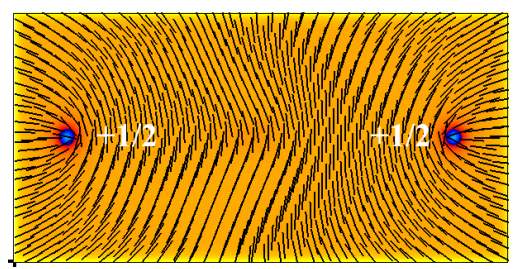

(1)

FiguRE 5. Defect dynamics with a strong anchoring (Dirichlet boundary condition). The color plot is the eigenvalue difference of eigenvalues on the $x y$ plane for $\mathbf{Q}+\frac{1}{3} \mathbf{I}$. The black line segments are the liquid crystal major director orientation on the $x y$ plane. (a-l) depict the orientation as well as the order parameter (the difference between the two eigenvalues whose corresponding eigenvectors are on the xy plane) at time $t=$ $1,2,5,6,7,10,15,16,17,18,19,20$, respectively. Initially, many defects are formed. But, only two defects survives in the long time simulation.

\section{Conclusion}

In this paper, a novel, second order in time, linear scheme is developed to simulate liquid crystal flow systems governed by a hydrodynamic $\mathbf{Q}$-tensor model. The new scheme demonstrates the following features. First of all, it is linear, which requires less computational cost to compute and is easy to implement. Secondly, it is second order and unconditionally energy stable in time. This allows us to use larger time steps while preserving the accuracy in numerical simulations. It extends our earlier work on a 


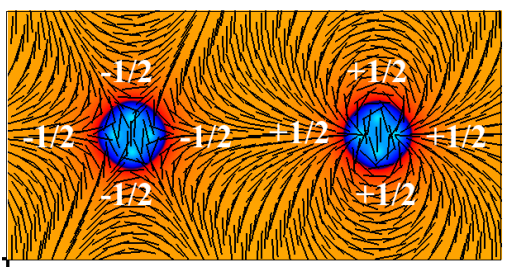

(a)

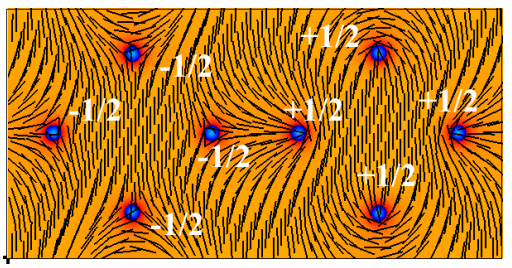

(d)

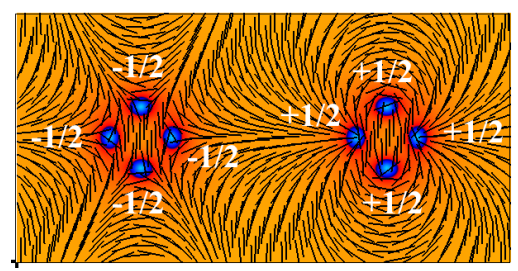

(b)

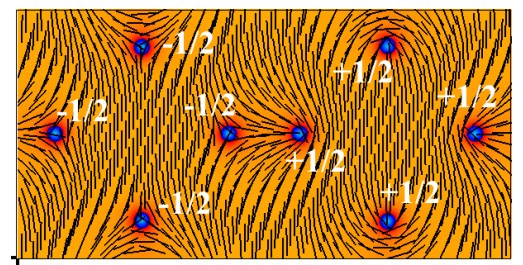

(e)

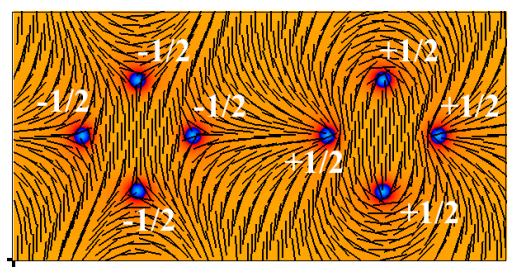

(c)

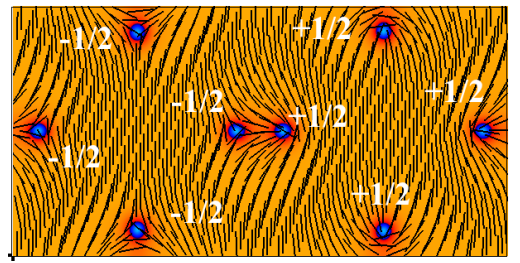

(f)

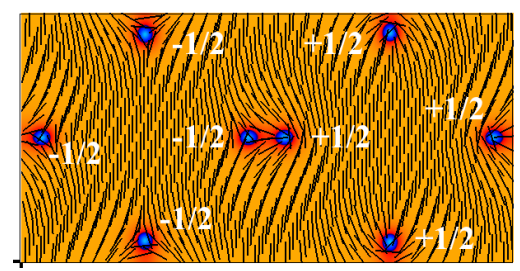

(g)

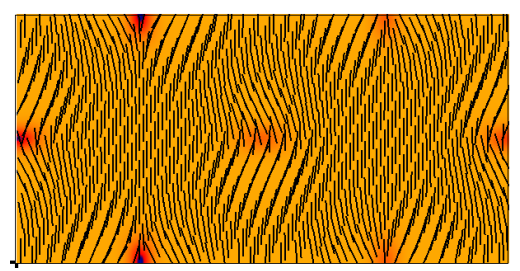

(h)

FiguRE 6. Defect dynamics with the soft anchoring (Neumann boundary condition). The color plot is the eigenvalue difference of eigenvalues on the $x y$ plane for $\mathbf{Q}+\frac{1}{3} \mathbf{I}$. The black line segments denote the liquid crystal major director orientation on the $x y$ plane. (a-h) depict the orientation as well as the order parameter at time $t=$ $1,2,5,10,12,15,16,18$. At the end of the simulation, all defects disappear.

similar model using a first order and a second order in time nonlinear scheme. This new scheme performs much better than nonlinear schemes which requires additional iterations. Several numerical examples are presented to demonstrate the usefulness of the new scheme and to exhibit a host of interesting defective dynamics in various liquid crystal flows. The energy quadratization methodology employed here can be extended to fairly general generalized hydrodynamic models governed by systems of dissipative partial differential equations.

\section{ACKNOWLEDGMENT}

Jia Zhao is partially supported by an ASPIRE grant from the Office of the Vice President for Research at the University of South Carolina. Xiaofeng Yang is partially supported by the U.S. National Science Foundation (NSF) under grant numbers DMS-1200487 and DMS-1418898. Yuezheng Gong is partially supported by China Postdoctoral Science Foundation through Grants 2016M591054. Qi Wang is partially supported by NSF grants DMS-1200487 and DMS-1517347, and NSFC-11571032.

\section{REFERENCES}

[1] R. Becker, X. Feng, and A. Prohl. Finite element approximations of the Ericksen-Leslie model for nematic liquid crystal flow. SIAM Journal of Numerical Analysis, 46(4):1704-1731, 2008. 


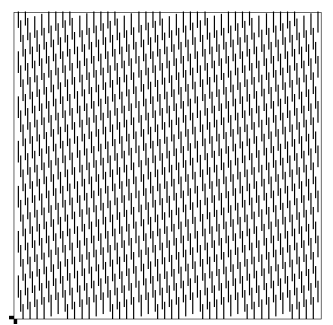

(a)

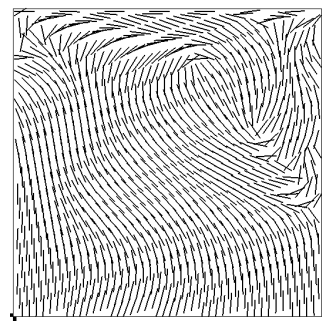

(e)

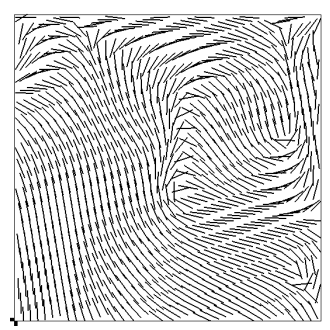

(i)

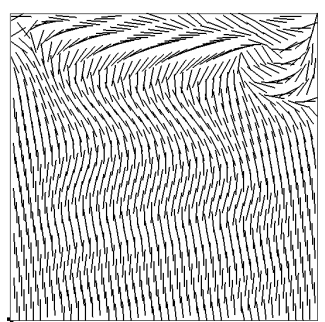

(b)

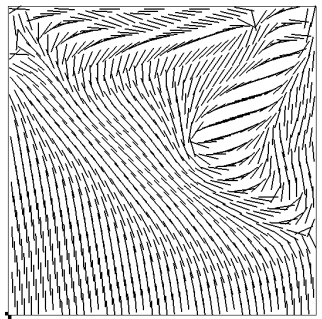

(f)

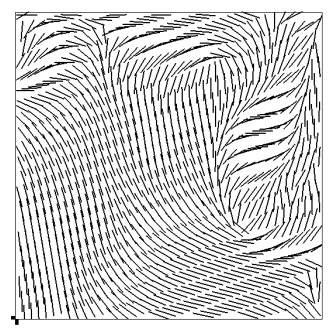

(j)

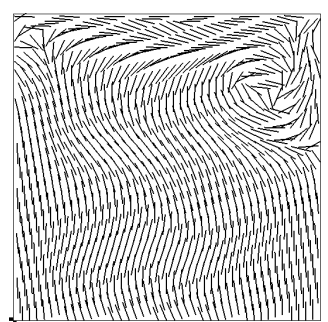

(c)

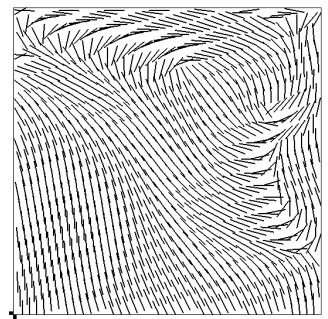

(g)

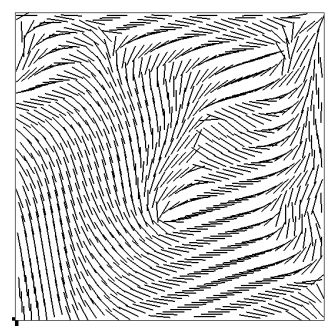

$(\mathrm{k})$

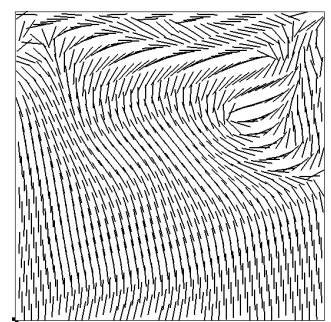

(d)

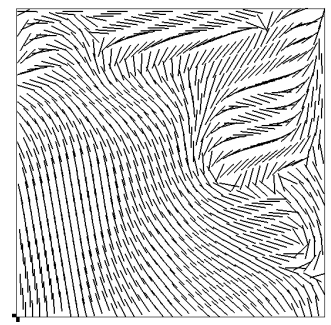

(h)

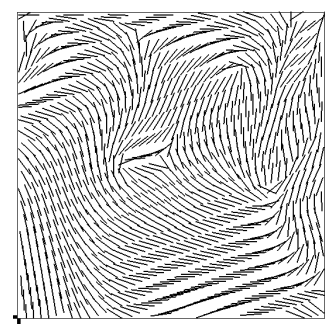

(1)

FIgURE 7. Liquid crystal dynamics in a cavity flow geometry subject to shear. The liquid crystal major director orientation on the $x y$ plane at time $t=$ $0,12,4,6,8,10,12,14,16,18,20,22$, respectively.

[2] A. N. Beris and B. Edwards. Thermodynamics of Flowing Systems. Oxford Science Publications, New York, 1994.

[3] C. Cavaterra, E. Rocca, H. Wu, and X. Xu. Global strong solutions to the full navier-stokes and q-tensor system for nematic liquid crystal flows in two dimensions. SIAM Journal of Numerical Analysis, 48(2):1368-1399, 2016.

[4] S. Chandrasekhar. Liquid Crystals. Cambridge University Press, 1993.

[5] X. Dai, J. Sun, and X. Cheng. Error estimates for an operator-splitting method for navier-stokes equations: secondorder schemes. Journal of Computational and Applied Mathematics, 231:696-704, 2009.

[6] P. G. de Gennes and J. Prost. The Physics of Liquid Crystals. Oxford University Press, 1993.

[7] I. Dierking, M. Ravnik, E. Lark, J. Healey, G. P. Alexander, and J. M. Yeomans. anisotropy in the annihilation dynamics of umbilic defects in nematic liquid crystals. Physical Review E, 85(2):021703, 2012.

[8] M. Doi and S. F. Edwards. The Theory of Polymer Dynamics. Oxford Science Publication, 1986.

[9] Masao Doi. Onsager's variational principle in soft matter. Journal of Physics: Condensed Matter, 23:284118, 2011.

[10] Q. Du, C. Liu, and X. Wang. Simulating the deformation of vesicle membranes under elastic bending energy in three dimensions. J. Comp. Phys., 212:757-777, 2005.

[11] J. L. Ericksen. Liquid crystals with variable degree of orientation. IMA Preprint Series 559, 1989.

[12] D. Eyre. Unconditionally gradient stable time marching the Cahn-Hilliard equation. Computational and mathematical models of microstructural evolution (San Francisco, CA, 1998), 529:39-46, 1998.

[13] J. Fan and T. Ozawa. Regularity criteria for a coupled navier-stokes and q-tensor system. International Journal of Analysis, 2013:718173, 2013. 


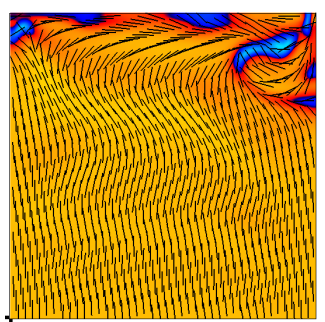

(a)

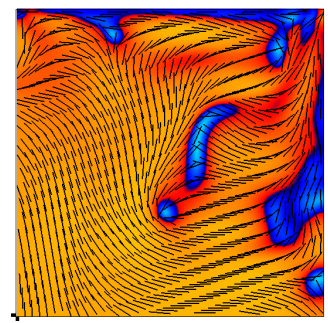

(e)

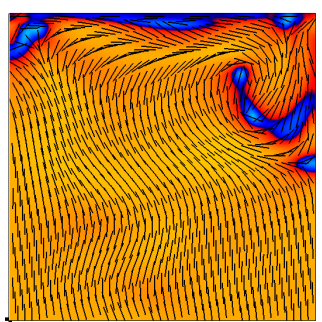

(b)

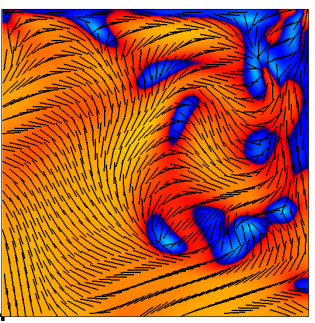

(f)

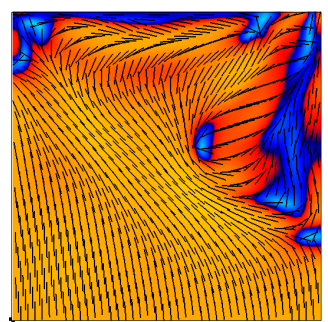

(c)

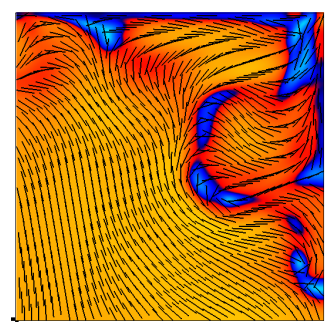

(d)

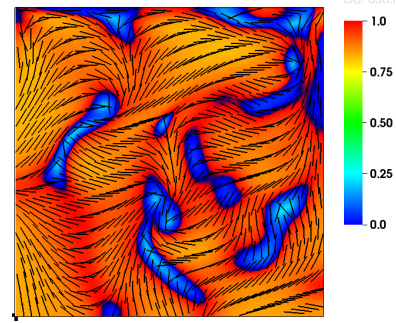

(g)

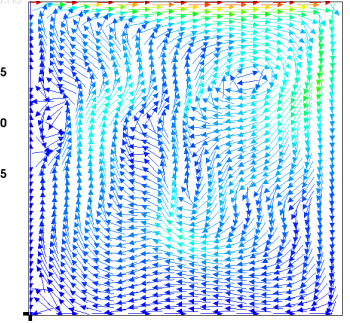

(h)

FIGURE 8. Liquid crystal dynamics in a cavity subject to shear. (a-g) The eigenvalue difference of eigenvalues on the $x y$ plane for $\mathbf{Q}+\frac{1}{3} \mathbf{I}$ and the major director orientation at time $t=2,5,10,15,20,25,30$, respectively, where the color map shows the eigenvalue difference. (h) The velocity field at $t=30$. More defects are created by the shear flow as time progresses.

[14] B. E. Griffith. An accurate and efficient method for the incompressible navier-stokes equations using the projection method as a preconditioner. Journal of Computational Physics, 228(20):7565-7595, 2009.

[15] J. L. Guermond and Jie Minev, P.and Shen. An overview of projection methods for incompressible flows. Comput. Methods Appl. Mech. Engrg, 195:6011-6045, 2006.

[16] F. Guillen-Gonzalez and G. Tierra. Second order schemes and time-step adaptivity for allen-cahn and cahn-hilliard models. Computers and Mathematics with Applications, 68(8):821-846, 2014.

[17] D. Han and X. Wang. A second order in time uniquely solvable unconditionally stable numerical schemes for CahnHilliard-Navier-Stokes equation. Journal of Computational Physics, 290(1):139-156, 2015.

[18] J.F. Joanny, F. Jülicher, K. Kruse, and J. Prost. Hydrodynamic theory for multi-component active polar gels. New Journal of Physics, 9:422, 2007.

[19] F. M. Leslie. The theory of flow phenomena in liquid crystals. Advances in Liquid Crystals, 4:1-81, 1979.

[20] F. Lin and C. Wang. Recent developments of analysis for hydrodynamic flow of nematic liquid crystals. Philosophical Transactions of the Royal Society of London Series A, 372:20130361, 2014.

[21] F. H. Lin. On nematic liquid crystals with variable degree of orientation. Communications on Pure and Applied Mathematics, 44:453-468, 1991.

[22] C. Liu and N. J. Walkington. Approximation of liquid crystal flows. SIAM Journal of Numerical Analysis, 37:725-741, 2000.

[23] C. S. MacDonald, J. A. Mackenzie, and A. Ramage. Efficient moving mesh method for q-tensor models of nematic liquid crystals. SIAM Journal of Scientific Computing, 37(2):215-238, 2015.

[24] R. Nochetto, S. W. Walker, and W. Zhang. A finite element method for nematic liquid crystals with variable degree of orientation. submitted, 2016.

[25] L. Onsager. Reciprocal relations in irreversible processes I. Physical Review, 37:405-426, 1931.

[26] L. Onsager. Reciprocal relations in irreversible processes II. Physical Review, 38:2265-2279, 1931.

[27] M. Paicu and A. Zarnescu. Energy dissipaiton and regularity for a coupled navier-stokes and q-tensor system. Archive for Rational Mechanics and Analysis, 203:45-67, 2012.

[28] T. Qian and P. Sheng. Generalized hydrodynamic equations for nematic liquid crystals. Physical Review E, 58(6):7475, 1998. 
[29] A. Ramage and A. M. Sonnet. Computational fluid dynamics for nematic liquid crystals. BIT Numer Math, 2015.

[30] A. D. Rey and M. M. Denn. Dynamical phenomena in liquid-crystalline materials. Annual Review of Fluid Mechanics, 34(1):233-266, 2002.

[31] J. Shen, C. Wang, X. Wang, and S. Wise. Second-order convex splitting schemes for gradient flows with EhrlichSchwoebel type energy: application to thin film epitaxy. SIAM Journal of Numerical Analysis, 50(1):105-125, 2012.

[32] J. Shen and X. Yang. Numerical approximation of Allen-Cahn and Cahn-Hilliard equations. Discrete and Continuous Dynamical Systems Series B, 28(4):1669-1691, 2010.

[33] J. Shen and X. Yang. A phase field model and its numerical approximation for two phase incompressible flows with different densities and viscosities. SIAM Journal of Scientific Computing, 32(3):1159-1179, 2010.

[34] R. Temam. Archive for rational mechanics and analysis volume 33, issue 5, january 1969, pages 377-385 sur l'approximation de la solution des équations de navier-stokes par la méthode des pas fractionnaires ii. Archive for Rational Mechanics and Analysis, 33(377-385), 1969.

[35] C. Wang, X. Wang, and S. M. Wise. Unconditionally stable schemes for equations of thin film epitaxy. Discrete and Continuous Dynamic Systems, 28(1):405-423, 2010.

[36] Q. Wang. Biaxial steady states and their stability in shear flows of liquid crystal polymers. Journal of Rheology, 41(943), 1997.

[37] Q. Wang. A hydrodynamic theory of nematic liquid crystalline polymers of different configurations. Journal of Chemical Physics, 116:9120-9136, 2002.

[38] Qi Wang. A hydrodynamic theory for solutions of nonhomogeneous nematic liquid crystalline polymers of different configurations. The Journal of Chemical Physics, 116:9120, 2002.

[39] M. F. Wheeler, T wick, and W. Wollner. An augmented-lagrangian method for the phase field approach for pressurized fractures. Computer Methods in Applied Mechanics and Engineering, 271:69-85, 2014.

[40] S. M. Wise, C. Wang, and J. S. Lowengrub. An energy-stable and convergent finite-difference scheme for the phase field crystal equation. SIAM Journal of Numerical Analysis, 47(3):2269-2288, 2009.

[41] S. Xu, P. Sheng, and C. Liu. An energetic variational approach for ion transport. Communications in Mathematical Sciences, 12(4):779-789, 2013.

[42] X. Yang, M. G. Forest, and Q. Wang. Near equilibrium dynamics and one-dimensional spatial-temporal structures of polar active liquid crystals. Chinese Physics B, 23(11), 2014.

[43] X. Yang, J. Zhao, and Q. Wang. Linear and unconditionally energy stable schemes for molecular beam epitaxial growth model based on energy quadratization methods. submitted, 2016.

[44] X. Yang, J. Zhao, and Q. Wang. Numerical approximations for the molecular beam epitaxial growth model based on the invariant energy quadratization. Journal of Computational Physics, in minor revision, 2016.

[45] X. F. Yang and J. Zhao. On linear and unconditionally energy stable algorithms for variable mobility cahn-hilliard type equation with logarithmic flory-huggins potential. submitted, 2016.

[46] X. F. Yang, J. Zhao, Q. Wang, and J. Shen. Numerical approximations of the three components phase field cahn-hilliard model. submitted, 2016.

[47] J. Zhao and Q. Wang. Semi-discrete energy-stable schemes for a tensor-based hydrodynamic model of nematic liquid crystal flows. Journal of Scientific Computing, 68:1241-1266, 2016.

[48] J. Zhao, Q. Wang, and X. Yang. Numerical approximations for a dendritic crystal growth model. International Journal for Numerical Methods in Engineering, In Press, 2016.

[49] J. Zhao, Q. Wang, and X. Yang. Numerical approximations to a new phase field model for two phase flows of complex fluids. Computer Methods in Applied Mechanics and Engineering, 310:77-97, 2016.

[50] J. Zhao, X. Yang, J. Shen, and Q. Wang. A decoupled energy stable scheme for a hydrodynamic phase field model of mixtures of nematic liquid crystals and viscous fluids. Journal of Computational Physics, 305:539-556, 2016.

[51] J. Zhao, X. Yang, and Q. Wang. Energy stable numerical schemes for a hydrodynamic model of nematic liquid crystals. SIAM Journal on Scientific Computing, 38(5):3264-3290, 2016. 